Review

\title{
Spectroscopy, Manipulation and Trapping of Neutral Atoms, Molecules, and Other Particles Using Optical Nanofibers: A Review
}

\section{Michael J. Morrissey ${ }^{1}$, Kieran Deasy ${ }^{2}$, Mary Frawley ${ }^{2,3}$, Ravi Kumar ${ }^{2,3}$, Eugen Prel ${ }^{2,3}$, Laura Russell $^{2,3}$, Viet Giang Truong ${ }^{2}$ and Síle Nic Chormaic ${ }^{1,2,3, *}$}

1 School of Chemistry and Physics, University of KwaZulu-Natal, Durban 4001, South Africa; E-Mail: morrissey@ukzn.ac.za

2 Light-Matter Interactions Unit, OIST Graduate University, 1919-1 Tancha, Onna-son, Okinawa 904-0495, Japan; E-Mails: kieran.deasy@ oist.jp (K.D.); mary.frawley @ oist.jp (M.F.); ravi.kumar@oist.jp (R.K.); eugen.prel@ oist.jp (E.P.); laura.russell@ oist.jp (L.R.); v.g.truong@oist.jp (V.G.T.)

3 Physics Department, University College Cork, Cork, Ireland

* Author to whom correspondence should be addressed; E-Mail: sile.nicchormaic@ oist.jp; Tel.: +81-98-966-1551.

Received: 31 May 2013; in revised form: 18 July 2013 / Accepted: 1 August 2013 / Published: 13 August 2013

\begin{abstract}
The use of tapered optical fibers, i.e., optical nanofibers, for spectroscopy and the detection of small numbers of particles, such as neutral atoms or molecules, has been gaining interest in recent years. In this review, we briefly introduce the optical nanofiber, its fabrication, and optical mode propagation within. We discuss recent progress on the integration of optical nanofibers into laser-cooled atom and vapor systems, paying particular attention to spectroscopy, cold atom cloud characterization, and optical trapping schemes. Next, a natural extension of this work to molecules is introduced. Finally, we consider several alternatives to optical nanofibers that display some advantages for specific applications.
\end{abstract}

Keywords: optical nanofiber; taper; evanescent field; cold atoms; atomic vapor; single particle detection; optical cavities; laser cooling; spectroscopy; whispering gallery resonators 


\section{Introduction}

Quantum mechanics plays a crucial role in the development and understanding of future technologies governed by quantum rules. The isolation of single atoms allows researchers to directly observe quantum properties of light-matter interactions. In recent years, researchers have been investigating the possibility of detecting, controlling, and manipulating quantum systems, such as cold atoms [1-4], trapped ions [5,6], and molecules [7], close to the surface of nanostructured devices. These devices include microcavities [8], atom chips [9], superconducting circuits [7], and optical nanofibers [1]. The interest in these devices primarily arises from their potential to offer methods by which single atoms/particles can be trapped, probed, and manipulated, thereby providing a useful tool for advancing quantum engineered devices.

The focus of this review paper is on the application of optical nanofibers (ONFs) [10-13] for the detection, manipulation, and trapping of laser-cooled atoms and the role they play in this, and related, research fields. Optical fiber sensors are a well-established tool [14] and, more recently, the versatility of ONFs as sensing tools $[15,16]$ for very small numbers of particles is also becoming evident. We begin by presenting the basic concept of the ONF and its fundamental properties. Section 2 introduces the ONF as a probe for either a cloud of magneto-optically trapped atoms or for atoms in a vapor. In these cases, the nanofiber can act as either a bright or dark probe with regard to the atoms. A bright nanofiber probe is generally used for absorption-type measurements, such as manipulation and trapping of atoms using light fields or for nonlinear optics with an atomic medium. In this case, lightusually tunable across an atomic transition of interest-is coupled into the optical fiber in the usual manner. This light propagates through the fiber, interacts with the surrounding medium at the narrowest region of the fiber (known as the waist), and then continues to propagate through the fiber until it is detected at the other end. Changes in the output intensity arise when interactions with the surrounding medium have occurred. In stark contrast, for a dark nanofiber, there is no initial light beam coupled into the fiber and any light detected at the output arises from fluorescence coupling from the surrounding medium into the nanofiber at the waist. The various techniques proposed to precisely trap and manipulate atoms are presented, as well as the atom-surface effects under such conditions. Aside from the progress in cold atom-fiber interaction experiments, ONFs have also found applications in sensing molecules, quantum dots, and nanodiamonds - areas that are reviewed in Section 3. In Section 4 some alternative systems for single atoms and molecules based on micro- or nanostructuring within the ONF itself are presented. The paper concludes with some comments on the future directions of this research field.

\subsection{Properties of an Optical Nanofiber}

An ONF is a circular dielectric waveguide with a diameter smaller than the wavelength of the light which propagates within it. Such an ONF can be realized at the waist of a biconical tapered optical fiber consisting of three distinct regions: (i) the normal fiber, (ii) the transition or taper region, and (iii) the waist, as illustrated in Figure 1. To fabricate such a device the same general fabrication technique is typically applied, involving the heating of an optical fiber to a molten state while elongating it to create a tapered optical fiber. Until now, a number of different heat sources have been 
used for this process, including a gas flame [16,17], a $\mathrm{CO}_{2}$ laser [18], a microheater [19], or an electrical strip heater [20]. Independent of the type of heat source, a pair of parameters are vital for determining the shape of the tapered region, along with the length and diameter of the ONF waist region [21,22]. These parameters are: (i) the size of the hot zone and (ii) the elongation length of the fiber. For this reason the flame brushing procedure is usually considered to be the preferred technique for incorporating optical nanofibers into cold atoms systems [16], since both of these parameters can be controlled with relative ease.

Figure 1. Schematic illustration of an optical nanofiber with a $125 \mu \mathrm{m}$ outer diameter and a core diameter of $4.4 \mu \mathrm{m}$. The narrowest region has a diameter in the range of hundreds of nanometers. Typical taper lengths are $\sim 6 \mathrm{~cm}$, with the waist region being a few $\mathrm{mm}$ in length.

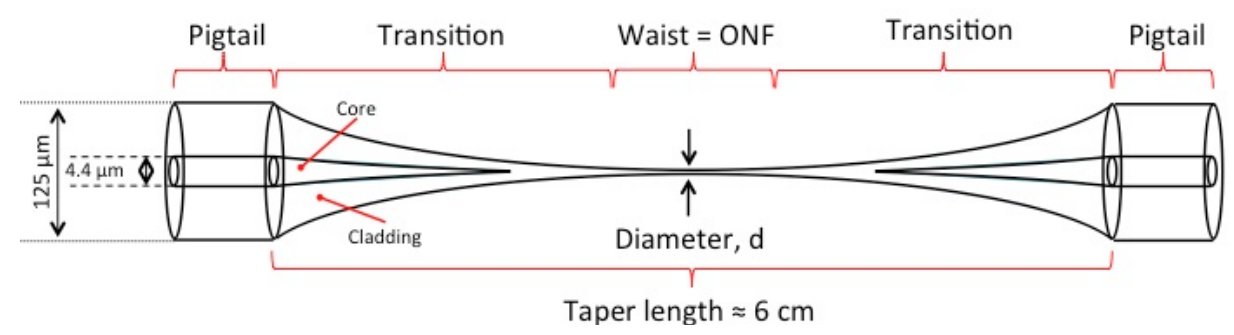

Another important factor in the fabrication of high performance ONFs is that optical losses due to the tapered regions should be kept to a minimum. To achieve this goal the adiabaticity criteria should be met [23]. These criteria take into account the changing profile of the fiber within the transition region and determine the best profile available. Assuming the diameter of the core and cladding decrease at the same rate, the core is reduced in size to the point where it has negligible influence on the guided modes within the ONF [24]. Thus, the guiding properties of the ONF are determined by the difference in the refractive indices between the cladding, $n_{\text {clad }}$, and the ambient environment, $n_{\text {amb }}$.

In many applications of ONFs the fiber should be single-mode, although interest in higher order mode propagation is increasing due to some advantages offered by modal interference for particle trapping [25-27]. The condition for a fiber to be single-mode is given by the $V$-number which depends on the fiber diameter, $d$, as well as the numerical aperture of the fiber, such that:

$$
V=\frac{k_{0} d \sqrt{n_{c l a d}^{2}-n_{a m b}^{2}}}{2}<2.405
$$

where $k_{0}=2 \pi / \lambda$ is the free space wave-number. This single-mode condition is illustrated in Figure 2, where only the fundamental, $\mathrm{HE}_{11}$, mode exists for values of the $\mathrm{V}$-number lower than 2.405. This condition is met experimentally by ensuring the ONF diameter is below the single-mode cut-off diameter.

Due to the sub-wavelength diameter of the ONF, the evanescent field [28] extends further into the optically rarer medium, such as a surrounding atom vapor, than in a conventional optical fiber. The light intensity on the surface can easily exceed the intensity inside the ONF if its diameter is small enough. Moreover, the polarization dependence of the decaying behavior in the evanescent field is more distinct in this case, and the field intensity varies azimuthally around the fiber, depending on the polarization of the propagating light. The enhancement of the evanescent field lays a favorable 
platform for the interaction of light with matter and also permits ONFs to be used for ultra-sensitive sensing applications.

Figure 2. A plot of the effective index of refraction, $n_{e f f}=\beta / k_{0}$, against $\mathrm{V}$-number for an optical fiber with cladding and core refractive indices as 1.4537 and 1.000 , respectively. $\beta$ represents the propagation constant. The vertical line indicates the boundary between single- and multimode guidance in the fiber $(\mathrm{V}=2.405)$.

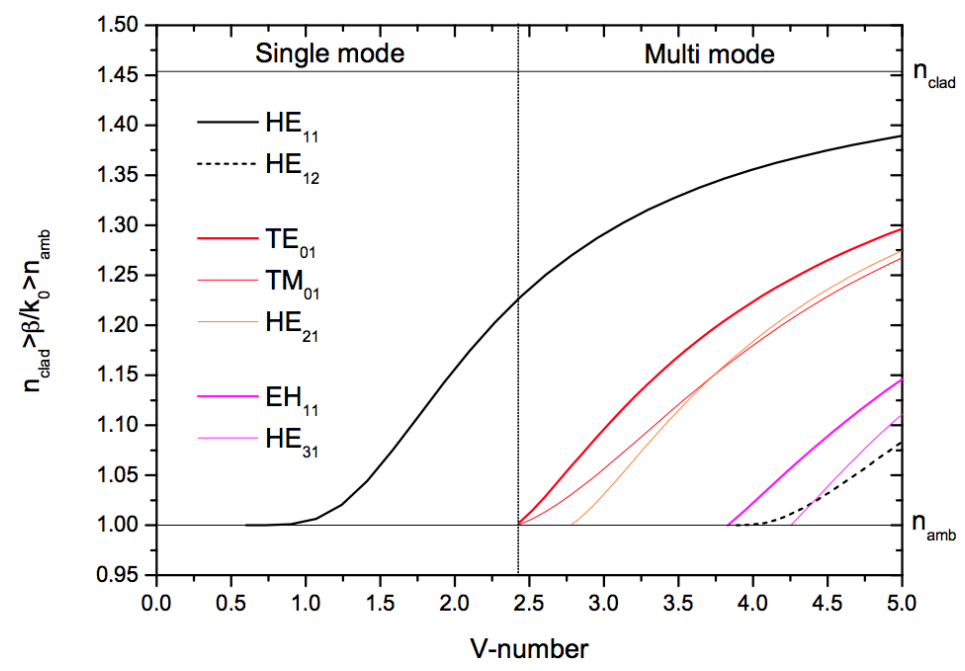

\subsection{Optical Nanofibers for Emerging Quantum Technologies}

In many areas of atomic physics, in particular for the development of quantum technologies, nonlinear optics provides a means of controlling the quantum states of light using atoms. While the focus of this paper is on sensing and manipulation of single or small numbers of particles around optical nanofibers, the overall motivation behind much of this work relates to emergent technologies in quantum networking using atoms and light, such as developing quantum logic gates via electromagnetically induced transparency (EIT) [29,30]. In any nonlinear system, in order to increase observable effects, there are two criteria which can be exploited, one of which is the nonlinearity of the medium and the other is the intensity of the light used. The optical depth of a medium, $O D$, is a unitless quantity that is related to the strength of the interaction between the medium and light, such that $O D=\eta \sigma L$, where $\eta$ is the density of the medium, $\sigma$ is the absorption cross-section of the atoms in the medium, and $L$ is the interaction length.

In an atomic system, $\eta$ is limited if one wants to avoid dephasing effects arising from atomic collisions. To maximize $\sigma$, the alkali atoms (such as $\mathrm{Cs}, \mathrm{Na}, \mathrm{Li}$, and $\mathrm{Rb}$ ) offer some of the highest absorption cross-sections available. Therefore, aside from choosing an alkali atom for observations of nonlinear effects, the obvious manner in which we can increase the optical depth is through increasing $L$, the interaction length. The optical nanofiber provides an ideal manner by which this can be achieved as the evanescent light field interacts with the surrounding medium over the taper length (typically several $\mathrm{mm}$ ). This is in contrast to the interaction that can be achieved using, for example, a very tightly focused laser beam (as is necessary to achieve a high intensity), where the interaction length is limited by the diffraction of the beam. As an example, a laser beam tightly focused to a waist of $2.3 \mu \mathrm{m}$ which interacts with a cloud of cold Cs atoms (transition wavelength $\sim 850 \mathrm{~nm}$ ) has a Rayleigh 
length of $\sim 20 \mu \mathrm{m}$ [31], orders of magnitude shorter than the few mm interaction length attainable with optical nanofibers.

The other parameter that can be optimized is the intensity of the laser used; ideally, the beam mode area should be proportional to the wavelength of light in order to maximize the available intensity. As mentioned above, for a free-space laser beam a high intensity can be achieved using a very tight focus, but this will reduce the interaction length, $L$, that can be achieved [31]. The optical nanofiber again provides an ideal platform for obtaining high intensities while maintaining a relatively long interaction length since the mode is confined within the evanescent field and very high intensities can be achieved for very low power inputs on the order of $\mathrm{mW}$ or less. The strong transverse mode confinement, in combination with the longer interaction length, would appear to suggest that nonlinear optics using optical nanofibers embedded in atomic systems may be an ideal tool for such studies. By increasing the fiber waist length through alternative profile shaping to the standard exponentially shaped taper [26] the system becomes even more favorable.

Aside from the advantages with regards to nonlinear optics, optical nanofibers are also extremely efficient devices for coupling fluorescence from surrounding particles, with up to $28 \%$ coupling efficiency achievable for a Cs atom near a $400 \mathrm{~nm}$ diameter ONF [32]. Coupling of light into subwavelength-sized structures is a major technical challenge, usually involving plasmonics [33,34], but the ONF influences the spontaneous emission from the atom, thereby leading to such high efficiencies in theory. It is also far easier technically to place atoms around a nanofiber than to place them into a fiber, as, for example, has been done by Ghosh et al. using Rb vapor in a photonic band-gap fiber (PBGF) [30]. The advantages offered by PBGF are similar to those for the ONF-a large interaction length, $L$, and confinement of the light to an area comparable to the atomic scattering cross-section, $3 \lambda^{2} / 2 \pi$, but the technical challenge of pumping the vapor into the fiber diminishes its functionality.

A drawback in using the optical nanofiber is the fact that surface interactions, such as van der Waals and Casimir-Polder, come into play and can affect both the resonance line shape and the central position in relation to free-space studies. A full understanding of the influence of surface interactions on line shape is essential in order to be able to distinguish this effect from others of interest and, while several theoretical studies have been conducted [35-37], further conclusive and substantiated experimental measurements are still required [38,39].

\section{Neutral Atoms}

The potential for using cold atomic ensembles in the evolution of quantum technologies is undisputed. However, it is clear that further advances in the precision control and manipulation of cold atoms are essential. Since the development of laser cooling of neutral atoms in the 1980's, many techniques have been successfully developed to trap [9,40-43], manipulate [44-50], and probe [51-53] cold atoms. Both trapping and manipulation of atoms can be performed using either magnetic fields or far-off resonance laser light, while probing is most conveniently performed with near- or on-resonant light. In recent years, ONFs have attracted considerable interest in the field of atom optics due to their ability to efficiently couple light and matter, thus having the ability to simultaneously trap, manipulate 
and probe neutral atoms [54]. There has also been a proposal on incorporating an ONF into an optical lattice to create small, cold atom samples with control over the final atom number [55].

Spontaneous emission from cold, neutral atoms that are sufficiently close to a subwavelength diameter fiber can couple into the guided modes of the fiber $[32,56]$. Due to multiple surface effects, which are described in Section 2.1, this emission can be strongly enhanced and the resultant fluorescence can be utilized to probe atoms in the vicinity of the ONF surface, as described in Section 2.2. The ONF can also be used as a detection device in a bright probe manner, whereby nearor on-resonant light propagating in the fiber creates an evanescent field, a portion of which is absorbed by the neutral atoms and this is covered in Section 2.3. These cold atom probing techniques are illustrated in Figure 3. To distinguish between the cases of fluorescence detection directly from a laser-cooled sample, as opposed to that where an additional free-space laser is incorporated into the setup, we will use the terms emission fluorescence detection for the former (Figure 3a) and laser-induced fluorescence (LIF) detection for the latter (Figure 3c). The absorption detection method, illustrated in Figure $3 \mathrm{~b}$ can also be extended to the detection of atoms in a vapor cell, as described in Section 2.4. By taking advantage of the evanescent field properties of the ONF, the combination of far-off-red and blue-detuned evanescent fields can be used to create two-color optical potentials, which can be used to trap and manipulate cold atoms. Variations of such techniques are described in Section 2.5.

Figure 3. Three techniques to detect neutral atoms using ONFs. (a) Emission fluorescence detection; (b) Absorption detection. Reprinted with permission from [57]. Copyright 2007 American Physical Society; (c) Laser induced fluorescence detection. Reprinted with permission from [58]. Copyright 2008 Institute of Physics. Note that in (c) an additional probe laser is used to excite the laser-cooled atoms, compared to the setup in (a).

(a) Emission fluorescence detection

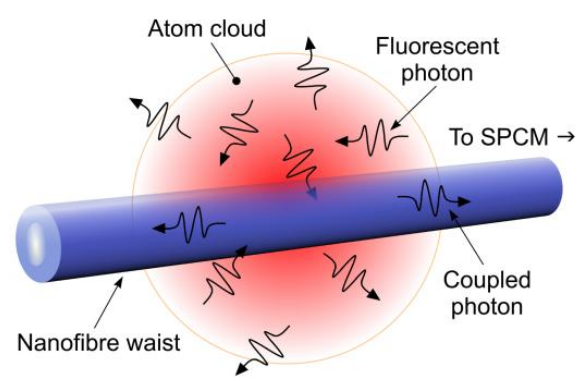

(b) Absorption detection

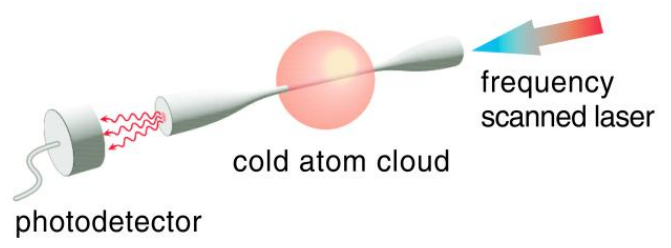

(c) Laser-induced fluorescence detection

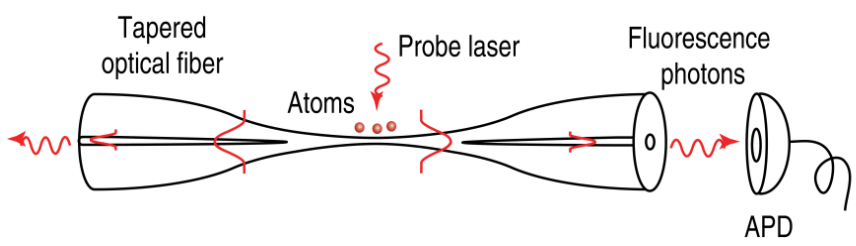




\subsection{Cold Atom-Surface Interaction}

The study of individual neutral atoms in the vicinity of dielectric surfaces has gained renewed interest in recent year [32,58-60]. Regarding ONFs, this is primarily due to their ability to manipulate atoms near their surfaces making them ideal for the development of devices in quantum optics and nanotechnology. In order to develop these technologies it is imperative to have a thorough understanding of how the presence of the ONF dielectric surface affects the behavior of the atom.

It is well known that the presence of any material body can substantially modify the spontaneous decay rate of an atom [32,61-64]. Spontaneously emitted photons close to the surface of the ONF can be radiated into space or they can be coupled into the guided modes of the ONF [65]. The presence of the ONF itself increases the coupling into the guided modes. The enhancement of the decay rates is largest when the atom is on the fiber surface and the effect reduces as the atoms are positioned further from the ONF surface. The decay rates not only depend on the position of the atom relative to the fiber surface, but also on the fiber size itself. The increase in coupling efficiency becomes significant when the diameter of the ONF is small in comparison with the radiation wavelength [32]. It also slightly varies for different magnetic sublevels. In a realistic system, a coupling efficiency of up to $28 \%$ of the spontaneously emitted photons can be achieved for a Cs atom near the surface of an ONF [32]. Such a high efficiency offers a promising technique for single atom detection.

The dominant electromagnetic interactions that take place in this context are the van der Waals [36] and Casimir-Polder forces [35]. The van der Waals force for ONFs can be viewed as an attractive force that pulls atoms towards the surface of the fiber and operates at distances $<\lambda / 10$ from the fiber surface, where $\lambda$ is the wavelength of the radiation. The Casmir-Polder force is also an attractive force, but only becomes significant at distances $>\lambda / 10$ from the surface. In the context of cold atoms, these forces have been measured for several surfaces [66,67] and can be exploited to create trapping potentials as discussed in Section 2.5.

The aforementioned atom-surface interactions cause a shift or perturbation in the spectral emission of excited atoms close to the surface of the ONF. Russell et al. [35] theoretically studied this effect by modeling the spectral properties of atoms whose spontaneous emission couples to the guided mode of the ONF, with the assumption that only the fundamental mode is excited. Here, the fiber was treated as a planer surface. It was determined that, for typical ONF diameters (200-600 nm), the fluorescence excitation spectrum exhibits a well-pronounced asymmetry with red-side broadening and a red-detuned shifting of the peak position caused by the van der Waals effect. The inclusion of the Casimir-Polder effect has minimal influence on the asymmetry of the line shape, but slightly reduces the red-shift of the peak position. Figure 4 shows the contribution from both the van der Waals and Casimir-Polder effects to the line shape in the presence of an optical nanofiber for three different fiber diameters. In addition, it was determined that the asymmetry becomes more pronounced for atomic ensembles that are tightly confined around the optical nanofiber. Later, Frawley et al. [37] considered the effect of the fiber curvature on the van der Waals interaction with an atom.

The predicted red-side-broadening effect has been experimentally observed by Sagué et al. [57] using absorption detection (see Section 2.3 for more details). Here, the asymmetries in the absorption profile of atoms interacting with the evanescent field of an ONF have been attributed to the van der Waals frequency shift. 
Figure 4. Frequency dependence of the fluorescence power from a Cs cloud coupled into an ONF, including contributions from the van der Waals and the Casimir-Polder effects. For this calculation a cloud radius of $400 \mathrm{~nm}$ has been used. The nanofiber radius is varied from $200 \mathrm{~nm}$ to $400 \mathrm{~nm}$ for the different plots. The solid line shows the expected free space line shape as a comparison. Note that all lines are normalized. Reprinted with permission from [35]. Copyright 2009 Institute of Physics.

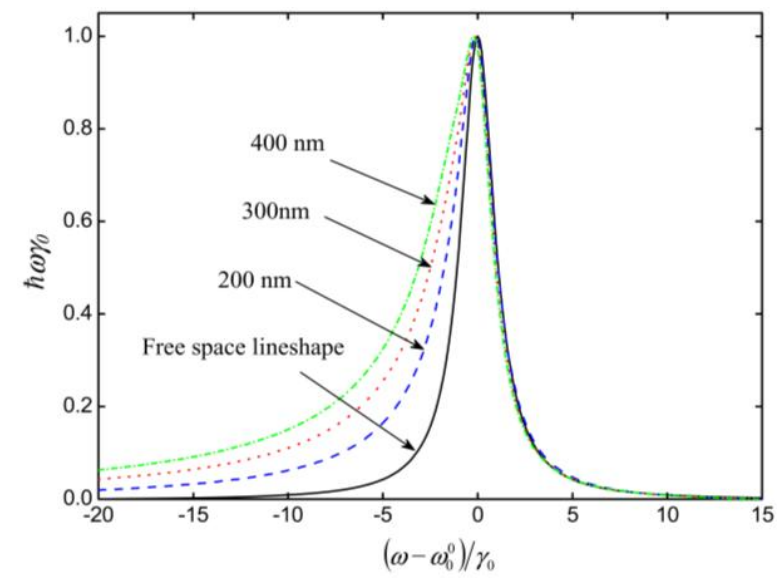

The red-shifted profile was also observed by Nayak et al. in 2008 [58], when Cs atoms were probed by scanning a laser perpendicular to the nanofiber through the closed-cycle transition $6 \mathrm{~S}_{1 / 2} \mathrm{~F}=4 \leftrightarrow 6 \mathrm{P}_{3 / 2} \mathrm{~F}^{\prime}=5$ (see Figure $3 \mathrm{c}$ for a schematic of the LIF detection setup). The LIF spectra were coupled into the guided modes of the fiber and a long red tail was observed, as represented by Trace A in Figure 5. This was initially attributed to the presence of atoms close to the surface and seemed to agree with theoretical predictions $[68,69]$.

Figure 5. Laser induced fluorescence spectra from $\mathrm{Cs}$ measured through an optical nanofiber for the closed-cycle transition, $6 \mathrm{~S} F=4 \leftrightarrow 6 \mathrm{P} F=5$. Traces A and B correspond to without and with the effect of violet laser irradiation, respectively. The violet irradiation was used to remove Cs that had adhered to the surface of the fiber. Reprinted with permission from [58]. Copyright 2008 Institute of Physics.

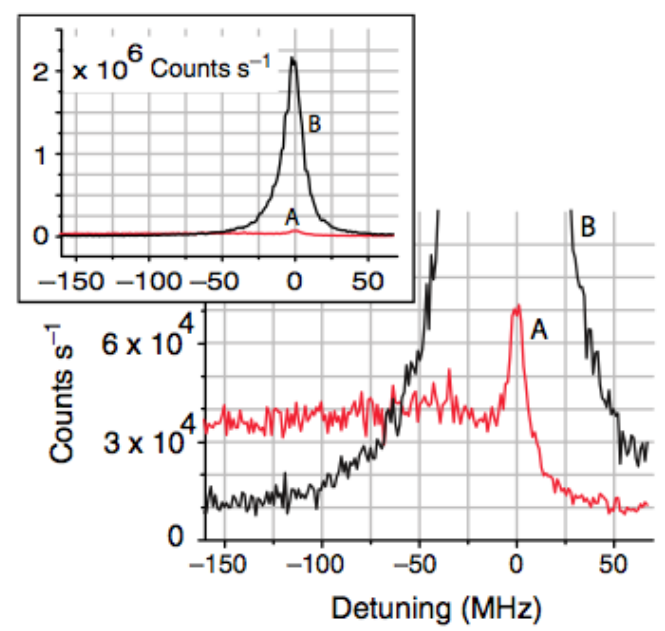


However, when Nayak et al. [70] performed a more systematic investigation of atoms in a surface-bound potential, this effect was found to be due to atoms on the surface itself rather than free, cold atoms. The authors first measured the LIF spectrum of cold atoms and investigated how this evolved over time for changing fiber surface conditions. The line shape of the spectrum changes from Lorentzian for free, cold atoms and, over time, the spectrum broadens to a large red tail line shape. This implies that, as time progresses, more and more atoms form bound-states with the fiber and induce a spectral line broadening effect. The narrow peak near the atomic resonance is due to the contribution from free atoms with the broad spectrum in the red-detuned side due to the bound atoms. The effect plays a crucial role in determining the surface conditions and leads to a reduction in the amount of laser induced fluorescence that is coupled to the guided modes of the fiber. In fact, it is suggested that the main source of the red tail is produced by room temperature atoms falling into the surface potential rather than the cold atoms themselves. Thus, the background density determines the speed at which atoms fall into the surface potential. The atoms can be removed from the bound potential with the aid of a violet laser and this can be used to control the surface conditions. In this manner the observed spectrum returns to its original state and the expected Lorentzian-shaped excitation spectrum is once again achieved. This provides a method by which the effect of surface conditions can be reduced or even eliminated.

\subsection{Fluorescence Detection of Cold Atoms}

The detection and quantitative analysis of cold atomic ensembles are essential analytical tools required in the development of quantum technologies. The simplest method for cold atom detection is fluorescence imaging, where the atoms are irradiated with resonant laser light and the resultant fluorescence from the atoms is focused on either a CCD camera or a photodiode. Using a combination of these devices, parameters such as size, number of atoms, and atom density profile can be easily determined with sufficient accuracy for most experiments. Dynamic properties of the experimental setup, such as loading rate, lifetime, and decay rate can also be determined.

For normal operational parameters of a magneto-optical trap (MOT), atoms in the cloud absorb light from the cooling laser beams exciting the atoms to a higher state. For atoms that are close to the surface of the ONF, a portion of the resultant spontaneous emission as the atoms decay back to the lower level can couple directly into the guided modes of the fiber waist [2,71]. With the aid of a single photon counter module (SPCM) attached to the end of the fiber, fiber-guided photons can be detected and the specific attributes of the cold atom system can be determined. The high coupling efficiency of fluorescence photons to the guided modes enables fluorescence measurements from a very small number of atoms. This is done while the atoms are continuously trapped in the MOT and thus a continuous real-time measurement is obtainable. This fluorescence detection scheme for cold atoms is illustrated in Figure 3a.

The detection technique was first implemented by Nayak et al. [38] whereby the coupling of the fluorescence from Cs atoms into the ONF was observed simply by monitoring the signal on the SPCM as the laser beams and magnetic field are sequentially switched (see Figure 6). An increase in photon count rate was observed when the MOT laser beams and the magnetic-field were switched on due to the spontaneous emissions from the cloud of Cs atoms coupling to the guided modes. From 
experimental parameters of the setup, it was determined that the effective number of atoms contributing to the signal at any given time was five. This accurately agreed with the photon count amplitude observed due to the presence of cold atoms around the fiber, indicating the potential of such devices for detection of a low number of atoms. Again, using this technique, the density profile, size and shape of the atomic cloud was determined by magnetically translating the cold atom cloud across the waist of the ONF and measuring the photon count rate as a function of cloud position. All the fiber fluorescence imaging results were compared to those achieved using standard fluorescence imaging techniques with photodiodes and CCD cameras and were found to be in good agreement.

Figure 6. Photon count rate through an optical nanofiber (diameter $400 \mathrm{~nm}$ ) in a Cs MOT setup. (1) MOT laser beams and magnetic fields are off; (2) MOT laser beams are on, magnetic fields are off; (3) MOT laser beans and magnetic fields are on. Reprinted with permission from [38]. Copyright 2007 Optical Society of America.

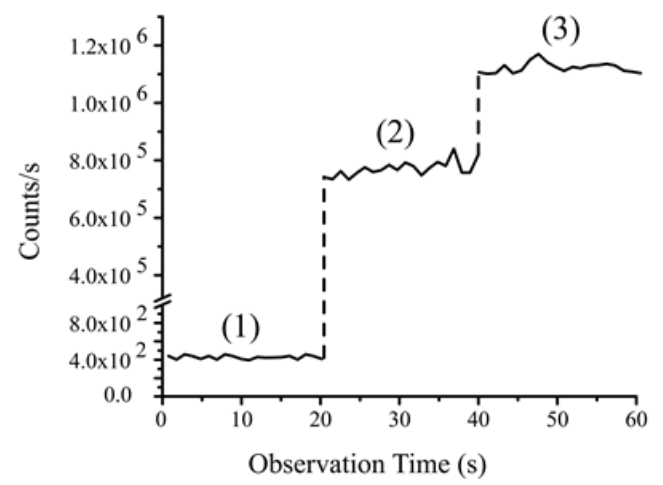

This emission fluorescence detection method was adopted by Morrissey et al. [72] where the technique was used to determine the dynamic loading rates and life-times of ${ }^{85} \mathrm{Rb}$ in a MOT, with a fiber of diameter $600 \mathrm{~nm}$. This was done by monitoring the coupled photons as a function of time as the MOT loaded from the background vapor and the photon signal decayed due to internal atom collisions when the atom source was switched off. The results were compared to those taken simultaneously using standard MOT measurement techniques and were found to be in good agreement. Using this same fiber fluorescence detection technique Russell et al. [3,73] measured ${ }^{85} \mathrm{Rb}$ temperatures above and below the Doppler limit of $144 \mu \mathrm{K}$ using two different methods, one being that of forced oscillations [3] and the other being that of release-recapture [73]. Observed temperature variation corresponds with the limits of normal MOT behavior. Again, good agreement was found between temperature measurements made using the optical nanofiber and conventional fluorescence imaging methods with a photodiode. Figure 7 shows a plot of temperature obtained for a cloud of ${ }^{85} \mathrm{Rb}$ using the forced-oscillation technique [3] for an optical nanofiber with a diameter of $700 \mathrm{~nm}$. Since the definition of cloud radius is required to determine the temperature using this technique, all three definitions commonly used in the literature $\left(1 / e^{2}, 1 / e\right.$ and FWHM) were tested in order to determine the viability of achieving sub-Doppler temperatures $(<144 \mu \mathrm{K})$ in the presence of the "hot" nanofiber. This was achieved when the cooling beams were significantly detuned from resonance. 
Figure 7. Atom cloud temperature as a function of detuning of the cooling laser beams for three standard definitions of cloud radius. The measurements were made using the forced oscillation technique and indicate that sub-Doppler temperatures $<144 \mu \mathrm{K}$ can be achieved in the presence of an ONF. Reprinted with permission from [3]. Copyright 2012 Institute of Physics.

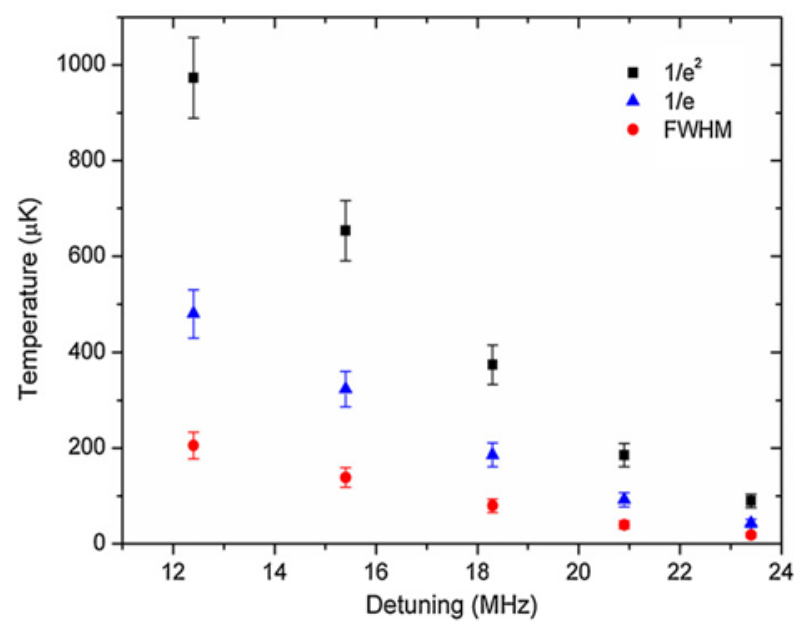

Nayak et al. [38] developed an alternative technique to measure the fluorescence of cold atoms with the aid of an ONF, which we've termed laser induced fluorescence detection to distinguish from the fluorescence methods already described. Once the cold atoms are prepared in the MOT, the trapping beams are switched off and, for a short period of time, the cold atoms are excited by a free-space, resonant probe beam. During this excitation period the atoms absorb from the probe beam resulting in the spontaneous emission of photons, some of which couple to the guided modes of the fiber and are counted by an avalanche photodiode (APD). The probe beam is then switched off and the MOT beams are switched on allowing the atomic cloud to reload from the background. This experimental setup is illustrated in Figure 3c. The observation is accumulated over many cycles. In this excitation fluorescence experiment the estimated atom number in the observation region is reduced by a factor of 70 when compared to direct emission fluorescence detection. This is due to the expansion of the atom cloud while the cooling beams are off during the excitation period. However, this method has the advantage of being insensitive to scattering from the irradiating light.

Using this technique, Nayak et al. [58] were able to measure the LIF spectrum and detected surface effects which have already been referred to in Section $2.1[38,70]$. This method can be extended to the detection of single atoms around the ONF [58]. In this case, the atom number is dramatically reduced by varying experimental parameters of the MOT. The excitation fluorescence that couples into the guided modes is split at the fiber output using a 50/50 nonpolarizing beam splitter and detected using two separate SPCMs. The photon correlation between the two channels is measured by performing a Hanbury-Brown and Twiss experiment. The photon coincidences clearly display anti-bunching effects, confirming the detection of single atoms using the ONF. Under these conditions the LIF spectrum was measured to further understand the atom behavior. For a low intensity of the probe beam the spectrum exhibits almost a Lorentzian shape, but is slightly asymmetric with a small red tail with no power broadening observed. This indicates that the spectrum is induced by free atoms in the vicinity of the ONF. However, there exists a small dip at the central peak. This dip is more pronounced for larger 
probe intensities and an additional broadening effect is observed. This dip was attributed to the mechanical effect due to scattering from the probe beam. It is worth noting that single atom behavior was only observed for clean fibers (after irradiation using a violet laser) — a further indication of the crucial role of surface interactions in such experiments.

\subsection{Absorption Detection of Cold Atoms}

Absorption imaging is a standard detection technique in cold atom physics whereby properties of the cold atom ensemble can be determined by measuring the absorption of a resonant light field. The strong evanescent field surrounding the waist of a sub-wavelength tapered fiber allows light-matter interactions with media surrounding the fiber, therefore facilitating in-fiber spectroscopy on the cold atom ensemble which surrounds the ONF. Given that the evanescent field decays exponentially from the surface, typically within a distance of $\lambda / 2 \pi$, this method can also be used to investigate surface interactions.

Work published in 2006 by Kien et al. [74] highlights the need for knowledge regarding the optical response of an atom in a resonant field which propagates along the surface of a fiber. The authors demonstrate that, for low field intensity of the evanescent field, when the atom is in close vicinity to the fiber surface, the scattered power can be up to $60 \%$ of the propagating power. When the fiber diameter is comparable to the wavelength of propagation, the light is mainly scattered into free-space, while scattering into the guided modes is weak. In the case of high propagation powers, a further increase in the propagation power will lead to a dramatic decrease in the scattering efficiency due to the saturation effect. This is a positive outcome as, even with small input powers, circulation intensities of the probe beam at the ONF waist are very high, as discussed in Section 1.2. This implies that a wide range of nonlinear effects are obtainable with minimal probe powers.

The first experimental demonstration of in-fiber spectroscopy of a cold atomic sample was published in 2007 by Sagué et al. [57]. Atoms are first captured and cooled in a standard MOT while the probe laser is switched off. The MOT cooling and repump laser beams, as well as the magnetic field, are switched off and the probe laser is switched on. During this time period the probe beam frequency is scanned around the atomic cooling transition, allowing the atoms to absorb the light propagating in the evanescent field, and the signal is measured on an APD. This measurement technique is illustrated in Figure $3 \mathrm{~b}$. A series of absorption spectra are shown in Figure 8 for three evanescent field intensities. The presence of a mode propagating through the fiber increases spontaneous emission by approximately $57 \%$ at the surface - an effect that had not been observed before to such an extent without a cavity. For probe powers larger than $100 \mathrm{pW}$, the line shapes are narrower than expected. This is explained by the effect of the light-induced dipole forces on the density of the atomic cloud. For distances smaller than $370 \mathrm{~nm}$, i.e., in the region that contains more than $75 \%$ of the evanescent field power, the largest integrated density of the atomic cloud is predicted in the case of zero detuning. For blue $(+3 \mathrm{MHz})$ and red $(-3 \mathrm{MHz})$ detunings, this integrated density is lowered due to the effect of the light-induced dipole forces. This results in reduced absorbance and leads to an effective line narrowing. The measured linewidths approach $6.2 \mathrm{MHz}$ for vanishing powers. This result exceeds the natural $\mathrm{Cs}_{2} \mathrm{D}_{2}$ linewidth in free space by almost $20 \%$. This broadening can be explained by surface interactions detailed in Section 2.1, i.e., the van der Waals shift of the Cs 
$\mathrm{D}_{2}$ line and the modification of the spontaneous emission rate of the atoms near the fiber. Both effects have the same magnitude and only their combination yields the very good agreement between the theoretical model and the experimental data. Other surface effects, such as the red-detuned-shifting of the center of the absorption profile, could not be measured due to the drift of the probe laser frequency. The authors conclude by highlighting that the subwavelength diameter fiber can be used to detect, spectroscopically investigate, and mechanically manipulate extremely small samples of cold atoms. On resonance, as little as two atoms, on average, coupled to the evanescent field surrounding the fiber and absorbed $20 \%$ of the total power transmitted through the fiber.

Figure 8. Line shapes obtained from an evanescent field around a nanofiber embedded in a laser-cooled sample of Cs. The measured linewidths approach the natural linewidth (6.2 MHz) for vanishing probe powers. Reprinted with permission from [57]. Copyright 2007 American Physical Society.

a)

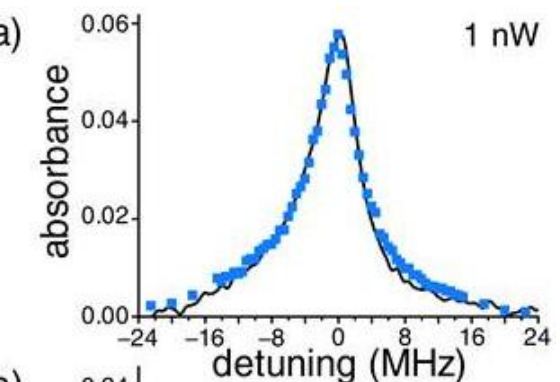

b)

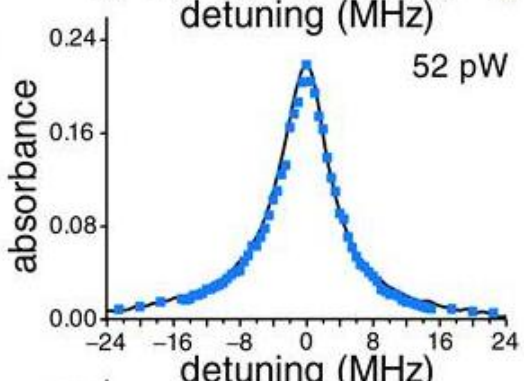

c)

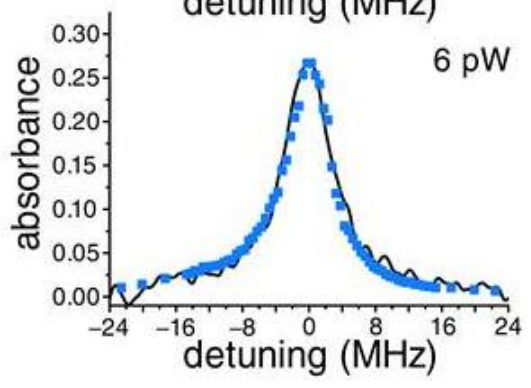

Due to the strong interaction between a few atoms and a relatively high intensity evanescent field, single and few atoms in the evanescent region can behave as an optically dense system. This is the subject of the work by Hakuta et al. [75]. They explore the atom-field interaction around a nanofiber using laser-cooled, Cs atoms and find that the atom/nanofiber interaction may open a new technique to trap single atoms without any external field. By using this trapping technique, they experimentally investigate small numbers of atoms by observing the fluorescence excitation spectrum.

Work published in 2008 by Nayak et al. [58] highlighted that, since an appreciable amount of propagating radiation is distributed in the evanescent region, single atoms in this region work as a good nonlinear medium due to their optical density. The photo-absorption spectrum is measured for a 
small number of atoms, revealing the possibility of realizing an optically dense system using hundreds of atoms. For example, photo-absorption through the nanofiber reaches about 50\% when one atom sits on the surface; if several atoms are prepared on the nanofiber surface, the system may become optically opaque. It should be noted that, when the atom is positioned away from the surface, the absorption becomes smaller, obviously, and one may need more atoms to realize an optically dense medium.

\subsection{Absorption Detection of Vapor Gas}

The concept of in-fiber spectroscopy for cold atoms can be easily extended to include atoms in a vapor. Due to the finite interaction time between the particle and the field compared to that for trapped atoms, transit-time broadening of the line shapes in spectroscopic measurements is observed $[39,76]$. A hot atom passes through the evanescent field in under 1 nanosecond, compared to a cold atom which has a transit time on the order of microseconds. A very insightful and early discussion on the effects of atom-light beam interaction time (i.e., transit time) in spectroscopy is contained in [76]. In 2008, Spillane et al. [77] discussed the observation of nonlinear interactions of a $\mathrm{Rb}$ vapor with an ONF-generated evanescent field using very low levels of input light. In fact, they were able to saturate the vapor using an input power level of $8 \mathrm{nW}$ as shown in Figure 9(left) and observed electromagnetically induced transparency (EIT), plotted in Figure 9(right).

Figure 9. (Left) Transmission spectrum for a nanofiber in a vapor cell for increasing probe powers $\left(2,4,8\right.$, and $10 \mathrm{nW}$ ) for the $\mathrm{Rb} \mathrm{D}_{2}$ transition; (Right) Saturated absorption spectrum with cross-polarized pump and probe beams for a Rb vapor cell (upper, blue) and a $\mathrm{Rb}$ nanofiber system (middle, green and lower, red) for the $\mathrm{D}_{1}$ manifold of ${ }^{85} \mathrm{Rb}$. The two side peaks correspond to the Doppler-free $F=3 \rightarrow F^{\prime}=2$ and $F=3 \rightarrow F^{\prime}=3$ hyperfine transitions. The center peak represents a V-system EIT signal. Reprinted with permission from [77]. Copyright 2008 American Physical Society.
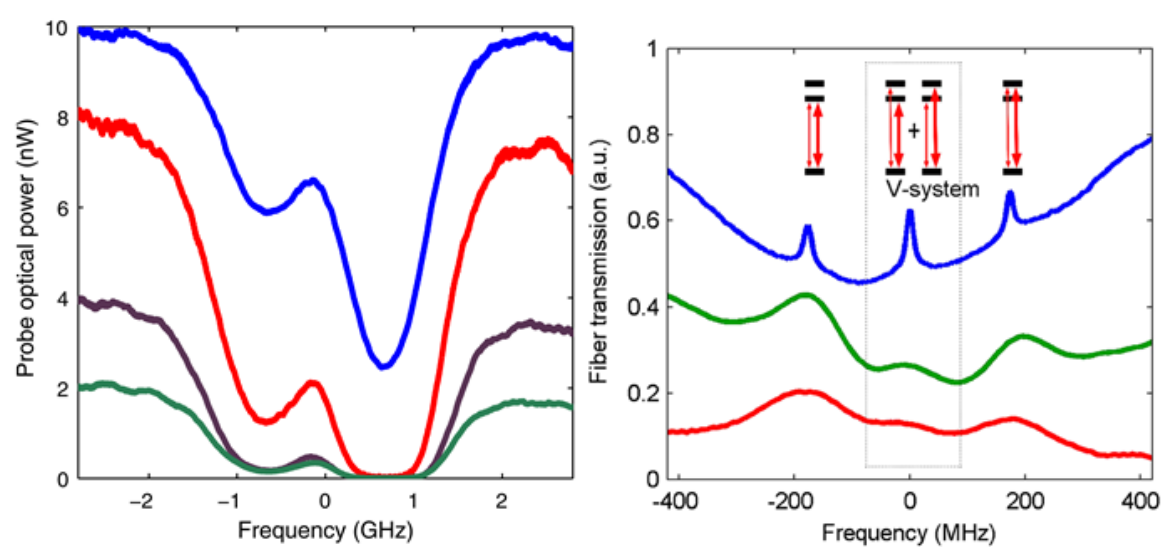

In 2009, Hendrickson et al. [78] observed transmission through a tapered fiber which was a nonlinear function of the incident power. This effect can also allow a strong control beam to change the transmission of a weak probe beam. This, once again, indicates that the ONF-atom interface can be used for nonlinear effects, such as EIT, slow light etc. For example, in 2010, Hendrickson et al. [79] published work about their observation of two-photon absorption in the ONF-vapor system using input 
light at levels below $150 \mathrm{nW}$. The transit-time broadening resulting from the fast atoms passing the nanoscale waveguide produces two-photon absorption spectra with sharp peaks that are very different from conventional line shapes (see Figure 10). Russell et al. [80] have since proposed using the ONF in a cloud of cold atoms for the demonstration of 1- and 2-photon absorption.

One issue associated with the use of the ONF in a vapor system is the degradation that the fiber transmission undergoes almost as soon as the vapor is introduced. This is a result of atoms adsorbing onto the fiber surface and has an impact on the lifetime of the fiber for experimental measurements. Recently [81], a systematic study has been conducted on the degradation effects, and methods of preserving the transmission by the incorporation of a microheater near the ONF have been demonstrated. This improves the future prospects of the ONF as a tool for technological advances in atom-based studies.

Figure 10. Resonant two-photon absorption in an ONF vapor system for Rb. The percent transmission of the $776 \mathrm{~nm}$ signal through the nanofiber is plotted as a function of its detuning from the upper atomic state. (a) $780 \mathrm{~nm}$ power level of $146 \mathrm{nW}$. (b) $780 \mathrm{~nm}$ power level of $726 \mathrm{nW}$. Reprinted with permission from [79]. Copyright 2010 American Physical Society.
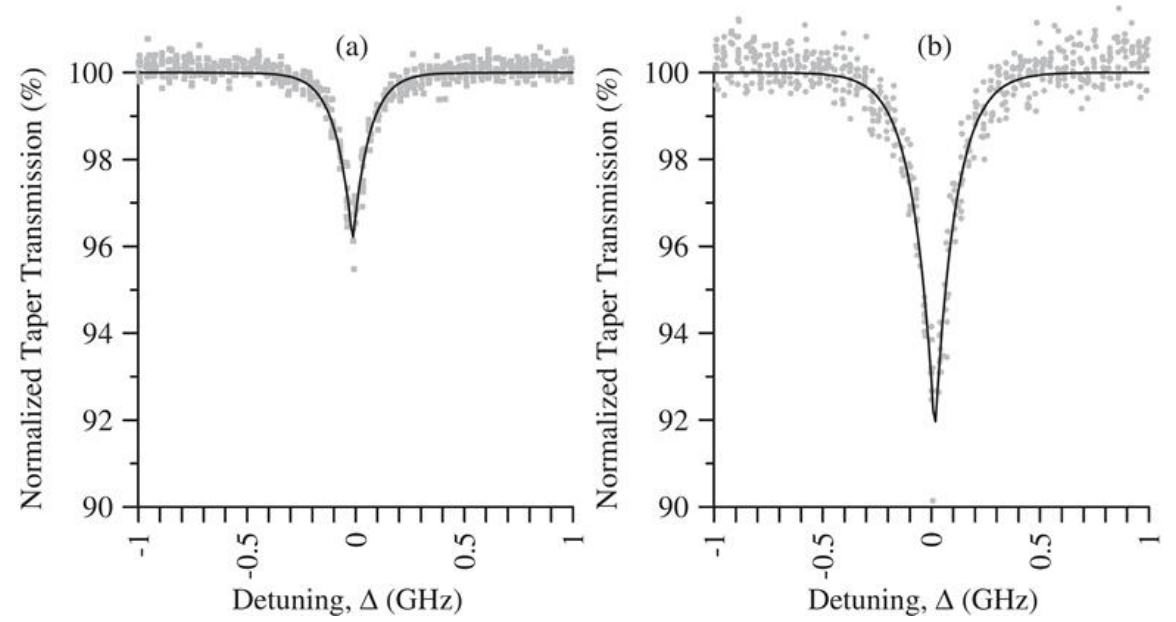

\subsection{Trapping of Neutral Atoms with ONFs}

As mentioned previously, the extension of the evanescent field from the ONF surface is very small and, thus, creates a high radial intensity gradient. This leads to a large gradient force on the atoms around the ONF in the transverse direction which can be utilized to create a dipole trapping potential. For red-detuned light, the optical force becomes attractive and blue-detuned light creates a repulsive force. By combining these forces along with the van der Waal's force, a dipole trapping potential for atoms around the ONF can be created. To date, several trapping schemes and configurations have been developed to trap, guide, and probe neutral atoms using ONFs, making it a powerful tool in this research area.

The first proposal regarding the trapping of neutral atoms around an ONF was written by Balykin et al. [82] and used a single frequency of light passing through the fiber. In this paper it was proposed to use a red-detuned evanescent field propagating through a subwavelength diameter ONF to 
create an optical potential attracting atoms towards the surface. For ONFs with a diameter two times smaller than the wavelength of propagating light, this attractive force can be counter-balanced by the centrifugal force of atoms moving in a circular motion around the TOF, thereby trapping the atoms close to the fiber surface.

Shortly after this theoretical publication, Le Kien et al. [83] proposed that neutral atoms can be trapped close to the surface of the ONF using two-color evanescent light fields. A simple schematic of the setup is shown in Figure 11. This proposed technique utilized a red-detuned light field, as well as the van der Waals force, to attract atoms towards the nanofiber, while a blue-detuned light field repels the atoms from the fiber. Due to the fact that the decay length of the evanescent field is wavelength dependent, by choosing the respective powers of the red- and blue- detuned beams a radial potential close to the nanofiber surface is created. If one or both fields are linearly polarized, two local minima are formed azimuthally around the fiber. This configuration allows atoms to be confined in two lines parallel to the fiber axis. If the input light fields are circularly polarized, a ring shaped potential is formed around the fiber resulting in atoms being confined to a cylindrical shell around the fiber.

Figure 11. Schematic representation of atom trapping and guiding around an optical nanofiber using two-color evanescent light fields. Reprinted with permission from [83]. Copyright 2004 American Physical Society.

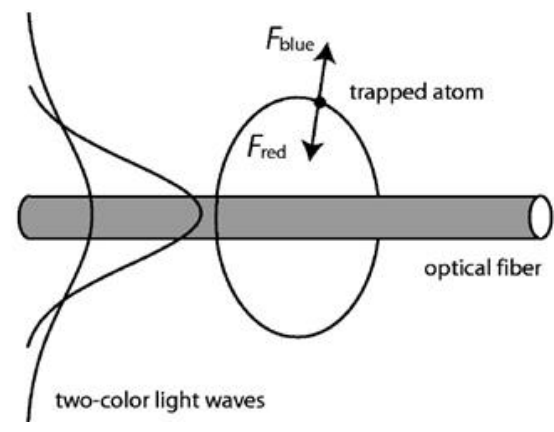

In 2010, Vetsch et al. [1] experimentally realized the two-color evanescent field setup to create a one dimensional optical lattice to trap Cs atoms around an ONF, work that was advanced by Dawkins et al. [84]. Two far-red-detuned lasers were counter-propagated through the ONF, producing an evanescent standing wave which creates an attractive force towards the fiber surface. This attractive force is balance by a single far-blue-detuned laser field. By choosing the correct power ratio between the red- and blue-detuned light fields, a minimum potential is achieved at a distance of a few $100 \mathrm{~nm}$ from the surface of the fiber, yielding trapping frequencies of $200,313,400 \mathrm{kHz}$ in the radial, axial, and azimuthal directions, respectively. The atoms are confined in trapping sites along the axis of the fiber by the standing wave and radially confined by the potential created by the red-and blue-detuned laser fields. All the laser fields were linearly polarized, thereby confining the atoms azimuthally. This experimental setup is depicted in Figure 12a, which includes an illustration of the lattice. Figure 12b shows a fluorescence image of the trapped atoms. The atoms were confined in a one dimensional optical lattice approximately $200 \mathrm{~nm}$ from the surface of the ONF, with each lattice site separated by $500 \mathrm{~nm}$. The average occupancy was 0.5 , limiting the trap to about 2,000 atoms per millimeter. When spectral properties of the atoms were investigated it was found that their linewidth was slightly larger than the atomic linewidth and this was apportioned to atom-surface interactions. This trapping 
technique can be easily adapted to create other configurations. Schneeweiss et al. [85] manually tuned the relative phase between the counter-propagating beams of the standing waves with the aid of acousto-optic modulators to demonstrate the optical transport of cold atoms along the nanofiber. Reitz et al. [86] proposed a double helix potential for the cold atoms using the same beam configuration as in [1], only with circularly polarized beams. The helical confinement arises from the beam intensity variations in the azimuthal direction [87]. Such a configuration would be extremely difficult to achieve using free space optics.

Figure 12. (a) Experimental setup of the ONF two-color atom trap. The blue-detuned running wave in combination with the red-detuned standing wave creates a trapping potential. A resonant laser is used for probing the atoms via the evanescent field. (b) Fluorescence image of the trapped atoms. Reprinted with permission from [1]. Copyright 2010 American Physical Society.

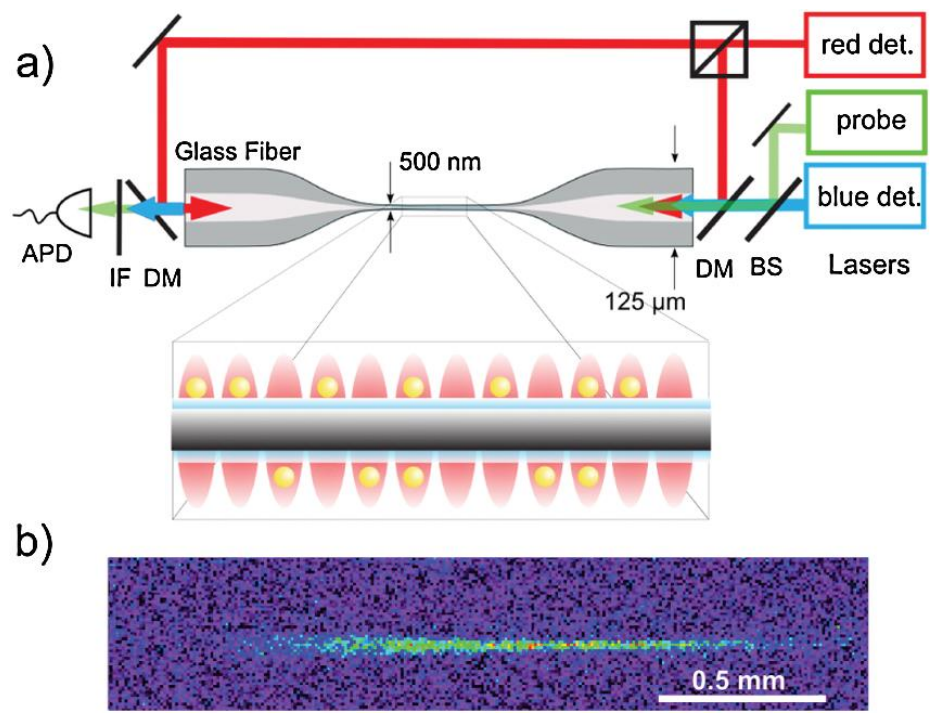

In 2012 Goban et al. [4] realized a two-color optical trap, which utilizes a so-called 'magic compensation' that traps the $6 \mathrm{~S}_{1 / 2}$ ground and $6 \mathrm{P}_{3 / 2}$ excited states of Cs. Similar to traps mentioned above, this configuration is created by two counter-propagating beams, which are red-detuned to the magic wavelength, $\lambda_{\text {red }}=937 \mathrm{~nm}$, creating a standing wave along the length of the ONF. This attractive force is balance by a second pair of counter-propagating beams blue-detuned to the magic wavelength, $\lambda_{\text {blue }}=686 \mathrm{~nm}$. Both beams are co-linearly polarized, resulting in a three-dimensional, state-insensitive, optical trap with approximately 224 atoms at a distance of $215 \mathrm{~nm}$ from the fiber surface. The power required for the red- and blue-detuned beams are 0.4 and $5 \mathrm{~mW}$ respectively, creating trapping frequencies of 199,273 , and $35 \mathrm{kHz}$ in the radial, axial, and azimuthal axes, respectively. The trapped atoms were analyzed using the absorption techniques mentioned in Section 2.3 where a probe beam propagates through the fiber, interacts with the trapped atoms in the ONF section of the fiber, and is measured at the other end by an APD. Due to the operation of the magic wavelength compensated trap, no appreciable shift in the transition frequency $(0 \pm 0.5 \mathrm{MHz})$ or broadening of the linewidth $(0.5 \pm 0.1 \mathrm{MHz})$ was observed. This is in contrast to the noncompensated trap [1], where a considerable shift in the transition frequency (13 MHz) and broadening of the linewidth (14.8 MHz broader than the natural linewidth) were observed. 
As an alternative to single or two-color trapping, Sagué et al. [25] proposed alternative techniques to trap atoms based on two-mode interference of a blue-detuned evanescent field of an ONF. The advantage of higher order mode interference for atom trapping around the fiber is that a higher level of control on the trapping sites can be achieved compared with one or two-color trapping. However, technical challenges associated with transmitting a higher order mode through the fiber with high survival rates still remain and solutions involving choice of core-to-cladding ratio of the pretapered fiber and nonexponential profile of the nanofiber are being pursued by several research groups $[26,27,88]$. The difference in phase velocities of two modes simultaneously copropagating through the fiber allows for the creation of a stationary evanescent interference pattern along the length of the fiber at a specific distance from the surface. Controlling the power distribution between the modes enables the modification of the evanescent field of each mode. This allows the creation of field minima where the two fields cancel due to destructive interference. The atoms are thus radially trapped due to the varying decay length of the modes, axially trapped due to the different phase velocities of the modes, and azimuthally trapped due to the polarization matching of the modes. Combining (for example) the $\mathrm{HE}_{11}$ and $\mathrm{TE}_{01}$ modes, or the $\mathrm{HE}_{11}$ and $\mathrm{HE}_{21}$ modes, results in two periodic arrays of traps on either side of the fiber in the axial direction. The site separation in the axial direction is determined by the beat length of the modes and the radial modes are axially offset from each other by an amount inversely proportional to the beat length. The $\mathrm{HE}_{21}$ and $\mathrm{TE}_{01}$ mode combination creates four axial arrays of traps, which are elongated due to the larger difference in the beat lengths. According to theory, the atoms are trapped about 100-200 nm from the surface of the ONF. The parameters of the trap are such as to achieve trap depths of $1 \mathrm{mK}$, a trap lifetime of $100 \mathrm{~s}$, with an initial kinetic energy corresponding to $100 \mu \mathrm{K}$. Depending on the mode combination the traps can be set up using powers of $25-50 \mathrm{~mW}$.

\section{Molecules and Other Particles}

Considering the aforementioned successes in using optical nanofibers for atom sensing, it is not surprising that they have also been used in experiments with molecules and other particles. This section gives a description of such advances, beginning with the use of nanofibers to examine spectroscopic properties of surface adsorbed particles. This offers a novel method for probing molecular photo-response, spectroscopic changes due to molecule-surface interactions, the dynamics of surface agglomeration, and thin-film formation. The section then develops to encompass advances in controlled microparticle, quantum dot, and nanodiamond deposition onto nanofiber surfaces. Such particles can then be site-addressed to obtain a myriad of spectroscopic and behavioral data through the fiber. Furthermore, quantum dot and (in particular) nanodiamond coupling to nanofibers have promising applications in the creation of fiber output single photon sources.

\subsection{Molecular Spectroscopy}

Nanofiber-based surface absorption spectroscopy of molecules was first examined by Warken et al. [89], who showed that the sensitivity of such a system is orders of magnitude higher than previous free-space techniques. Due to the their spectral sensitivity to molecular surface arrangement, 3,4,9,10-perylenetetracarboxylic dianhydride molecules (PTCDA) were selected for experiment. These 
crystals were heated below a nanofiber, and sublimated molecules were adsorbed onto its surface. Absorption spectra were obtained through the fiber for various deposition times (0.5 to $2.3 \times 10^{7}$ molecules), showing clear vibronic progression. Additionally, molecular condensation and agglomeration over time resulted in transmissional line shifts, allowing the authors to investigate the post-deposition film evolution on the nanofiber surface. The noted molecular agglomeration subsequently received further study [90], where the dynamics of the system under ambient and ultrahigh vacuum (UHV) conditions were compared, and significant reduction of molecular mobility in UHV was observed.

A similar deposition method was later used to excite and detect PTCDA fluorescence spectra through a nanofiber [91]. Interestingly, the absolute peak positions were shifted relative to those obtained from solution spectra. The authors associate this with the interaction of the molecules with the fiber surface, and also highlight the non-negligible contribution of self-absorption of fluorescence photons to recorded spectra. This should be minimized, or compensated for, to retrieve the expected mirror symmetry between absorption and fluorescence spectra. Nonlinear experiments have also been conducted with nanofibers, demonstrating two-photon excited fluorescence measurements of adsorbed Rhodamine 6G (Rh6G) molecules. For this experiment, a solvent-dripping technique was developed to extend the applications of surface adsorption spectroscopy to a larger variety of molecules [90]. The aforementioned surface adsorption techniques are highly applicable for modeling systems of organic thin-film growth and - as they are entirely fiber-based - may be used for remote spectroscopic studies. Crucially, they facilitate recording of both absorption and fluorescence spectra for a given molecular surface coverage, and pave the way for nanofiber self-absorption free fluorescence spectroscopy on individual surface-adsorbed molecules.

Optical nanofibers have also been used in saturation absorption spectroscopy studies of acetylene $\left({ }^{12} \mathrm{C}_{2} \mathrm{H}_{2}\right)$ molecules in a chamber at 200 Pa pressure [92]. Passing an infrared (IR) pump beam through the nanofiber and retroreflecting it as a probe beam, the spectra of the P9 transition were obtained with a narrow saturated signal. The dependence of the saturation parameter on nanofiber diameter was also calculated and optimized. Due to the fact that lowering the gas pressure can reduce the pressure broadened width, longer ONF lengths are desirable for such studies. Wiedemann et al. [93] recently presented a novel application for nanofibers where organic photochromic molecules show a reversible light-induced change of their absorption spectra and are thus ideal candidates for optical switching studies using nanofibers. Low concentrations of SpiroOH molecules which photoswitch between transparent and colored when exposed to UV and white light were drip-coated onto the nanofibers State-switching was then induced by simultaneously coupling light from a UV LED through the fiber. Absorbance spectra confirmed the switching mechanism; this effect can be seen as a time progression in Figure 13a,b. Photobeaching, photodestruction, and cyclability of the molecules were also investigated using the optical nanofibre and the authors indicate that switching speeds $(0.025-1 \mathrm{~Hz})$ could be increased by orders of magnitude by propagating tailored laser pulses through the fiber. 
Figure 13. Absorbance spectra of spiroOH on a nanofiber, with white light and additional UV-light exposure. All molecules are initially in the transparent state; (a) UV illumination increases the absorbance up to a stable point in the photostationary state; (b) The absorbance decreases after the UV exposure has stopped. Reprinted with permission from [93]. Copyright 2012 Optical Society of America.

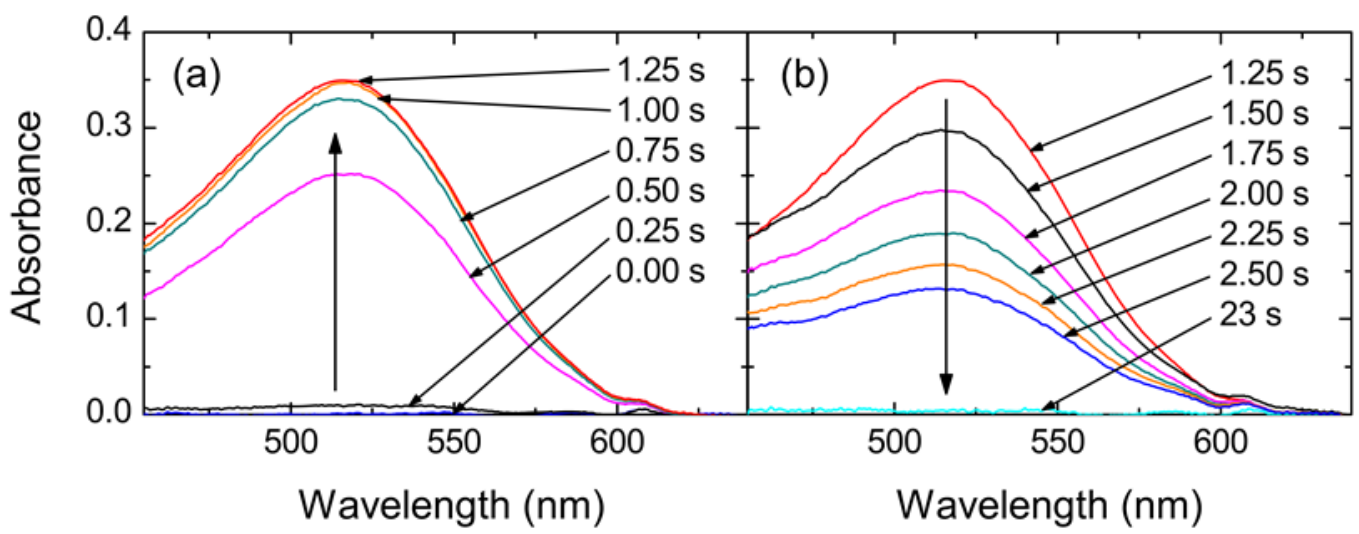

As with neutral atoms, bringing single particles into contact with the evanescent field of a nanofiber has obvious potential for both sensing and spectroscopic studies. Such a technique was demonstrated by Gregor et al. [94], who introduced individual charged particles to the surface of a nanofiber via a segmented linear Paul trap. The individual fluorescent dye-doped polystyrene beads were excited with the aid of a microscope objective and preselected before being brought to the fiber surface with a success probability close to $100 \%$. The authors compared the fluorescence spectra achieved using the microscope objective to that obtained through the fiber and calculated a seven-fold overall increase in detected fluorescence using the nanofiber. They also used FDTD simulations to relate the dip observed in fiber transmission following particle introduction to the system to the number of particles in an incident cluster. This noncontact electrospray injection method can facilitate the study of any type of charged particle which can be brought into suspension.

\subsection{Quantum Dots and Nanodiamonds}

Optical nanofibers were first used for single quantum dot photoluminescence spectroscopy by Srinivasan et al. [95]. A single layer of InAs quantum dots (QD) was embedded in an $\operatorname{In}_{0.15} \mathrm{Ga}_{0.85} \mathrm{As}$ quantum well, grown at the center of a GaAs waveguide. To increase accessibility to the QDs and reduce the overall QD number, microdisk cavities of diameter $d=2 \mu \mathrm{m}$ were fabricated from the material and cryogenically cooled to $14 \mathrm{~K}$. The authors compared the QD emission spectra obtained from objective lens pumping to those obtained by pumping and collecting via nanofiber coupling to the disk. This showed a 25 fold increase in the collected power through the fiber, without coupling to whispering gallery modes in the disk. Spatial mapping and single QD photon collection was demonstrated by scanning the nanofiber over the surface of the disk. Focusing on photon collection efficiency, Davanco et al. [96] subsequently modeled optical nanofiber coupling to single emitters embedded in thin dielectric membranes. FDTD simulations were run for the fiber and membrane as a composite system with associated supermodes, and the modified spontaneous emission rate of an embedded quantum dot was modeled as a two-level atom. The fiber collection efficiencies calculated 
for both vertically and horizontally oriented dipoles amount to up to 30\%, which exceed those obtainable with a high NA objective by an order of magnitude. To further increase the efficiency of such a system, they modeled the optical nanofiber coupling to a quantum dot embedded in a suspended semiconductor channel waveguide [97], predicting that up to $70 \%$ of the dipole's emission can be collected by the nanofiber in this system. The authors subsequently theoretically investigated nanofiber coupling to individual emitters bound to the surface of thin dielectric membranes via polymer, sol-gel, or crystalline hosts [98], again yielding high collection efficiencies.

Simplifying the aforementioned systems to quantum dots attached directly to nanofibers, Garcia-Fernandez et al. [90] dripped CdSe QDs dissolved in heptane onto a nanofiber waist. Adsorbed dots were calculated to number $3 \times 10^{5}$, and absorption and fluorescence spectra were recorded through the fiber. In a similar theme to above, an observed shift in the fluorescence maximum was attributed to surface interactions with the nanofiber. Yalla et al. [99] subsequently progressed this line of inquiry to observe fluorescence and emission properties of single quantum dots. To achieve this, they deposited CdSeTe (ZnS) QDs at $20 \mu \mathrm{m}$ intervals along a nanofiber using a sub-picoliter needle dispenser, with a positioning accuracy of $5 \mu \mathrm{m}$. Deposition sites were individually excited using an inverted microscope, as illustrated in Figure 14(left). Photon correlations were measured from one fiber pigtail, clearly showing anti-bunching behavior, and QD blinking was also observed. Single-step and double-step blinking corresponded to one and two QDs on site, respectively, where the probability of depositing a single QD using this method was estimated at $60 \%$ and fluorescence photon coupling rates into fiber guided modes were calculated. The saturation behavior of the QDs relative to excitation intensity was also addressed (see Figure 14(right)). The channeling of fluorescence photons into the nanofiber modes was studied, highlighting that the total channeled efficiency should take into account the efficiency of the QD itself [100]. To accurately measure this, the authors simultaneously recorded the photon count rates through both the guided and radiation modes of a nanofiber. QD sites were individually excited through an objective lens and the guided fluorescent photons were detected through the fiber pigtails. To factor in radiation mode guidance, fluorescent photons were also collected by the objective. Photon emission rates into both guided and radiation modes were calculated by analyzing photon-count rate histograms. By factoring in the light transmission parameters for both mode paths, the maximum channeling efficiency of $(22.0 \pm 4.8) \%$ agreed with the theoretical predictions [32,64]. It is expected that a photon channeling efficiency of higher than $90 \%$ could theoretically be obtained by incorporating a cavity structure on a nanofiber [99].

Other work related to photon coupling from nanoemitters into optical nanofibers is contained in Fujiwara et al. [101]. Here, the authors coupled $(7.4 \pm 1.2 \%)$ of the photons emitted from single $\mathrm{CdSe} / \mathrm{ZnS}$ quantum dots into an ONF of diameter $300 \mathrm{~nm}$. A schematic of the experimental setup is given in Figure 15(left). The authors were able to obtain scanning images of the QD on the fiber down to single quantum dot resolution and made second order photon correlation measurements. Clear anti-bunching was observed with a $g^{(2)}(0)$ value of 0.96 obtained. Their results are presented in Figure 15(right), including the dependence of the photon counts on the excitation laser intensity. 
Figure 14. (Left) Fluorescence counts depicted through a nanofiber as each QD deposition site is individually excited through a microscope objective; (Right) Observed fluorescence count-rate for increasing intensities at positions 3, 4, 5 and 7 in (a), showing QD saturation. Reprinted with permission from [99]. Copyright 2012 Optical Society of America.
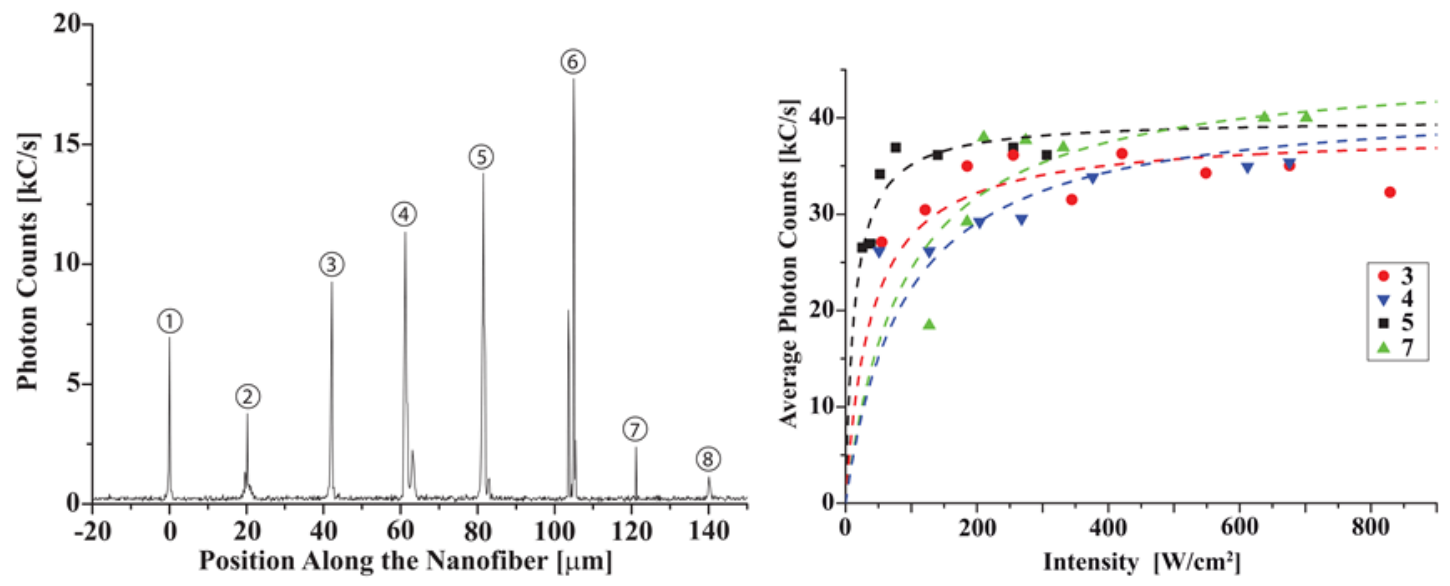

Figure 15. (Left) Schematic illustration of the setup. Note that the detection method was changed from APDs to a spectrometer, depending on the experiment. (Right) Scanning images of quantum dots on the ONF using (a) an objective and (b) an ONF. The quantum dot is next to the white arrow. (c) Second order photon correlation measurements. (d) Photon count from the quantum dot as a function of excitation laser intensity. Reprinted with permission from [101] . Copyright 2011 American Chemical Society.

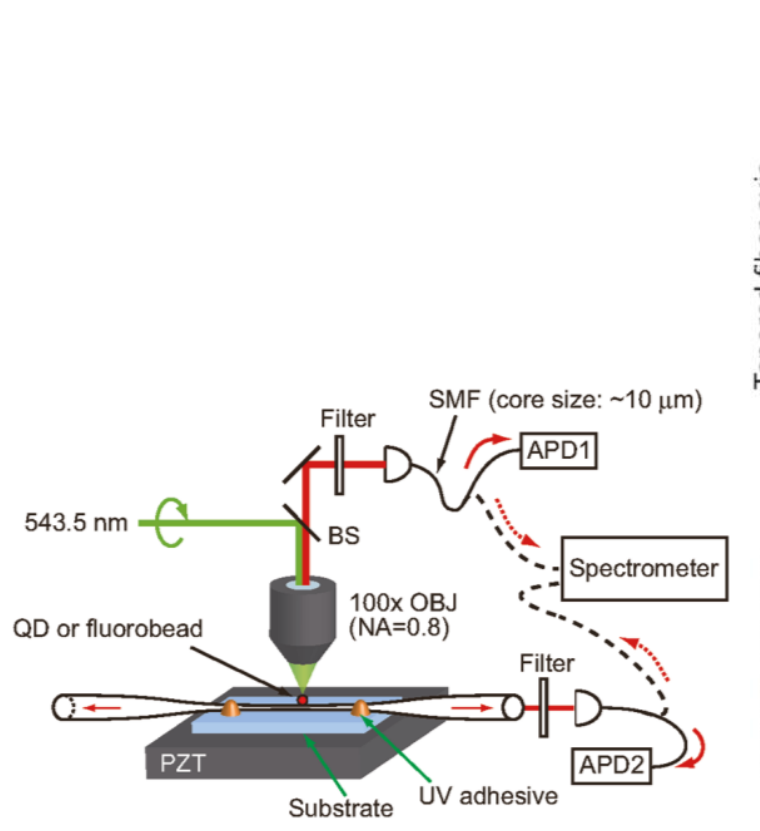

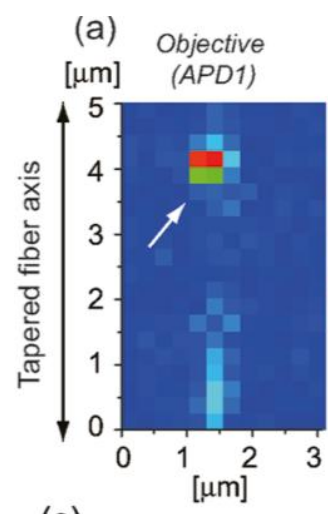

(c)

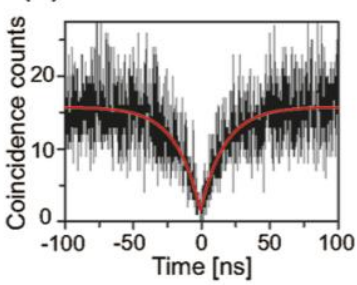

(b) Tapered fiber

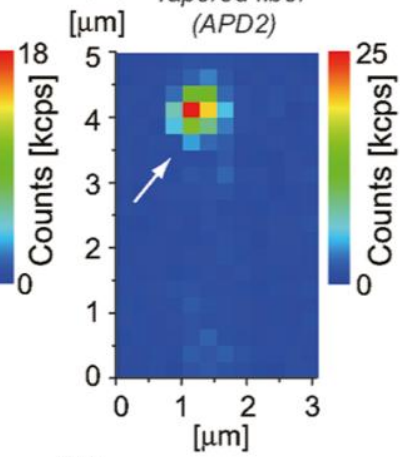

(d)

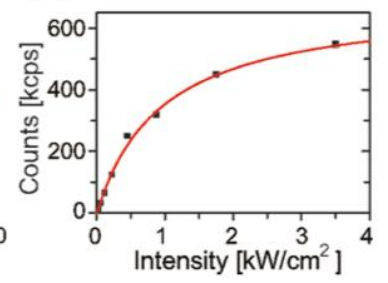

As QDs typically suffer from blinking and photobleaching, their potential as stable single photon sources is questionable. Nanodiamonds with nitrogen-vacancy-centers which are free from these difficulties have been deposited onto optical nanofibers by a dip-coating and translation technique. This scheme facilitates real-time estimation of the attached diamond numbers through fiber transmission monitoring [102]. Individual diamonds were illuminated through a microscope objective. 
Red-shifted fluorescence spectra of these nanodiamonds were obtained through the fiber, and auto- and cross-correlation measurements showed anti-bunching of photons, again indicating that the light came from a single photon emitter. Combining nanofibers with unblinking color centers, such as nanodiamonds, could lead to fiber-coupled, on-demand single photon generation [103,104]. The techniques described above also offer exciting possibilities for nanofiber detection and spectroscopy of biomolecules [105].

\section{Alternative Techniques}

The development of optical cavities has been of great interest in research for many years due to the significant enhancement effects and detection sensitivity offered by these devices [106]. With improved fabrication techniques various types of optical microcavities have been investigated [107-112]. Such microcavities have proven to be a productive platform for experiments with such effects as strong coupling [113] and the Purcell effect [114] being demonstrated. For detection purposes, microcavities are extremely sensitive devices, arising from the low optical mode volume and long interaction times available due to the high-Q factors [106,115], thereby enabling the detection of single atoms [113] and single molecules [116].

Typically, for optical microcavities, the trend has been to use optical nanofibers to achieve coupling of light into and out of the cavity modes [113,117-121]. Due to the advantages offered by cavities for detection, a movement from these two components to a simpler system where the cavity is directly incorporated within the tapered optical fiber itself is underway. An example of this comes in the form of bottle resonators [18,122-124], where the taper region itself is deformed to create a resonator, which generally still requires an external taper to couple light into the cavity. This shows how the tapered fiber can itself be used as a cavity. More direct examples of cavities within the fiber come in the form of fiber Bragg gratings (FBGs), for which much theoretical work has already been done on the interaction of a few/single atoms close to the nanofiber cavity [103,125-129].

The fabrication of such FBGs has been developing in recent years, from micron scale fibers down to nanometer scale fibers. For this, three fabrication techniques are used most frequently: (i) ultraviolet irradiation [130-133], (ii) focused ion beam (FIB) milling [104,134-137], and (iii) femtosecond laser irradiation [138-141]. Each technique has advantages and disadvantages with quite possibly the most work to date concentrating on the FIB technique, see Figure 16 for an example [104]. More recently, however, there has been success using femtosecond laser ablation to create highly ordered photonic crystal structures on a nanofiber, see Figure 17 [141]. Such nanofiber cavities offer to be promising devices for the detection of very low numbers of atoms.

One problem with fixed cavities in the nanofiber region is that of tuning the cavity to useful wavelengths for detection purposes. This can be achieved, for example, by straining the fiber [142]. Other resonators which can be fabricated directly from tapered optical fibers and may avoid this problem are those where the fiber is looped upon itself. Examples of this are microcoils [143-145], microfiber knots [146,147], and microfiber loops [148,149]. With these cavities, different tuning opportunities may be available [150-154]. For future possibilities using optical nanofibers, it has already been shown that polarization maintaining fiber can be tapered to micron sizes, whilst still maintaining the polarization of the coupled light [155]. Such control and maintenance of polarization 
within the fiber may be of use when probing polarization sensitive states of atoms, or utilizing polarized confined modes within an optical cavity.

Figure 16. Scanning ion microscope (SIM) image of a nanofiber Bragg grating fabricated using the FIB milling technique. The fiber diameter is $\sim 560 \mathrm{~nm}$. The grating period is $\sim 360$ $\mathrm{nm}$, with each groove having a depth of $\sim 100 \mathrm{~nm}$ and width of $\sim 150 \mathrm{~nm}$. The scale bar represents $1.0 \mu \mathrm{m}$. The image was taken at $60,000 \times$ magnification used in the SIM, but the scale bar gives the correct scaling for this image. Reprinted with permission from [104]. Copyright 2011 Optical Society of America.

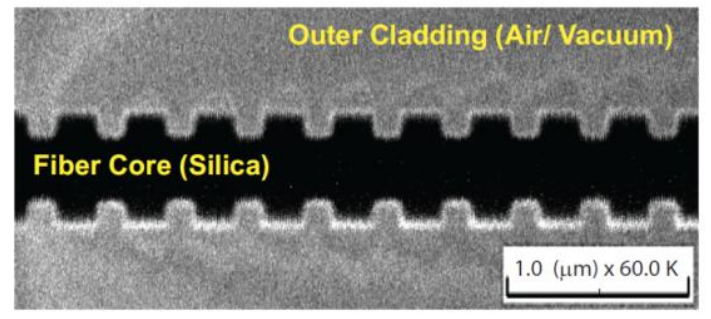

Figure 17. (a) Scanning electron microscope image of a structured nanofiber fabricated using single-shot irradiation of the ONF by a femtosecond laser beam. Inset: Enlarged view. Periodic nanocrater structures are observed on the shadow side of the nanofiber. (b) Cross-sectional image of a nanocrater measured by tilting the nanofiber. Reprinted with permission from [141]. Copyright 2013 Optical Society of America.

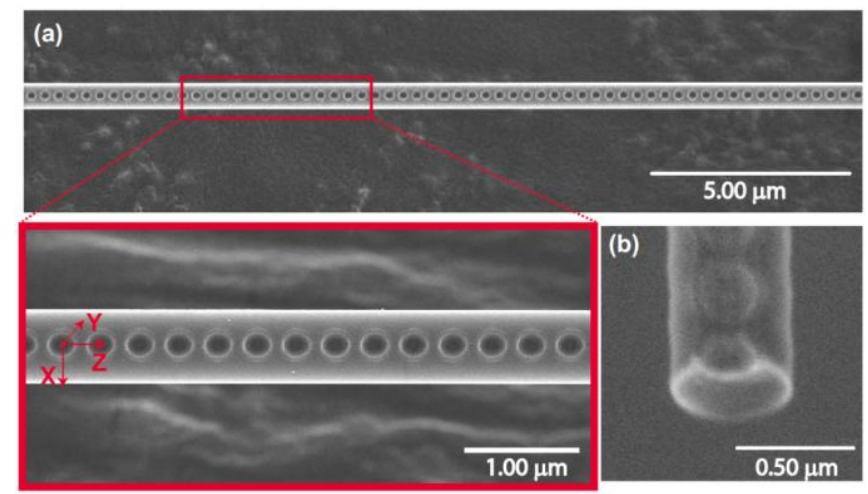

\section{Conclusions/Outlook}

The systematic fabrication of ONFs has now become readily available, with several techniques already developed to achieve a smooth surface profile with low transmission losses and high mechanical strength, making them ideal for the researcher to utilize in the development of quantum optical devices. In this article, we have reviewed the state-of-the-art schemes that utilize ONFs for ultra-sensitive detection, trapping, and manipulation of atoms and molecules. Due to the high coupling efficiency of atoms into the guided modes of the fiber, as well as the significant fraction of photons that can be absorbed from the evanescent field, ONFs provide an interface whereby the spectral properties of the atoms close to the surface of the fiber can be investigated. The ONF has the inherent ability to suppress ambient scattering of light, while maintaining the high coupling efficiencies of atoms. This allows the ONF to operate as a nondestructive detector to determine characteristics of 
atom clouds such as temperature, size and shape, as well as dynamic properties such as loading and decay times. Such techniques can have many future applications for nondestructive characterization of atomic ensembles. Based on the same principal, the ONF also acts as a sensor device for the detection of few atoms, as well as single atoms, with a high signal-to-noise ratio. These techniques have shown that ONFs have promise for numerous applications in quantum technologies, where detection of small numbers of atoms will be essential.

Due to the fact that atoms are detected close to the surface of the fiber, the detection schemes can facilitate fundamental research into physical phenomena, such as the van der Waal and Casimir-Polder forces, which are otherwise more difficult to observe. These surface effects become important when considering the long-term performance or the degradation of the ONF in cold atom systems, whereby atoms become bound to the surface decreasing the performance of the ONF over time. This destructive effect can be reversed with the aid of a specific laser frequency passing through the fiber, thereby extending the lifetime of the ONF.

The unique properties of the ONF and its evanescent field allow various trapping schemes to be implemented. The configurations that have already been realized rely on two separate frequencies of light, with specific powers and polarizations, to create local optical lattice sites close to the surface of the ONF. The traps provide strong confinement of single atoms in each lattice site. The atoms trapped in the sites can be translated by controlling the relative detuning of the standing waves, giving the ONF the ability to simultaneously, trap, probe, and manipulate single atoms. This demonstrates the versatility and functionality of the ONF and paves the way for future ONF experiments, such as entanglement via photon exchange.

Although the focus of this review has been on the utilization of ONFs with cold atoms, the methods and techniques can be easily extended to systems other than atoms. Molecules can be detected by performing surface absorption spectroscopy via the ONF. This has proven to be a more sensitive method when compared to free space techniques. In addition, for the use of an ONF as an interface with cold atoms, the structure of the ONF itself can be modified to incorporate devices such as grating and cavities. This micro/nanostructuring of the ONF opens the path for multiple possibilities regarding the future of optical nanofibers as tools in the field of atom optics.

\section{Conflicts of Interest}

The authors declare no conflict of interest.

\section{References}

1. Vetsch, E.; Reitz, D.; Sagué, G.; Schmidt, R.; Dawkins, S.T.; Rauschenbeutel, A. Optical interface created by laser-cooled atoms trapped in the evanescent field surrounding an optical nanofiber. Phys. Rev. Lett. 2010, 104, 203603.

2. Das, M.; Shirasaki, A.; Nayak, K.P.; Morinaga, M.; Le Kien, F.; Hakuta, K. Measurement of fluorescence emission spectrum of few strongly driven atoms using an optical nanofiber. Opt. Express 2010, 18, 17154-17164.

3. Russell, L.; Deasy, K.; Daly, M.J.; Morrissey, M.J.; Nic Chormaic, S. Sub-doppler temperature measurements of laser-cooled atoms using optical nanofibres. Meas. Sci. Tech. 2012, 23, 015201. 
4. Goban, A.; Choi, K.S.; Alton, D.J.; Ding, D.; Lacroute, C.; Pototschnig, M.; Thiele, T.; Stern, N.P.; Kimble, H.J. Demonstration of a state-insensitive, compensated nanofiber trap. Phys. Rev. Lett. 2012, 109, 033603.

5. Brownnutt, M. Private Communication, Innsbruck, Austria, March 2013.

6. Brownnutt, M. Ions and Fibres: A Target Rich Environment. In Proceedings of Optical Nanofiber Applications (ONNA2013), Okinawa, Japan, 1-7 June 2013.

7. Andre, A.; Demille, D.; Doyle, J.M.; Lukin, M.D.; Maxwell, S.E.; Rabl, P.; Schoelkopf, R.J.; Zoller, P. A coherent all-electrical interface between polar molecules and mesoscopic superconducting resonators. Nat. Phys. 2006, 2, 636-642.

8. Aoki, T.; Dayan, B.; Wilcut, E.; Bowen, W.P.; Parkins, A.S.; Kippenberg, T.J.; Vahala, K.J.; Kimble, H.J. Observation of strong coupling between one atom and a monolithic microresonator. Nature 2006, 443, 671-674.

9. Reichel, J. Microchip traps and Bose-Einstein condensation. Appl. Phys. B Las. Opt. 2002, 74, 469-487.

10. Tong, L.M.; Gattass, R.R.; Ashcom, J.B.; He, S.L.; Lou, J.Y.; Shen, M.Y.; Maxwell, I.; Mazur, E. Subwavelength-diameter silica wires for low-loss optical wave guiding. Nature 2003, 426, 816-819.

11. Tong, L.; Lou, J.; Mazur, E. Single-mode guiding properties of subwavelength-diameter silica and silicon wire waveguides. Opt. Express 2004, 12, 1025-1035.

12. Tong, L. Brief introduction to optical microfibers and nanofibers. Front. Optoelectron. China 2010, 3, 54-60.

13. Tong, L.M.; Zi, F.; Guo, X.; Lou, J.Y. Optical microfibers and nanofibers: A tutorial. Opt. Commun. 2012, 285, 4641-4647.

14. Lee, B. Review of the present status of optical fiber sensors. Opt. Fiber Tech. 2003, 9, 57-79.

15. Moar, P.; Huntington, S.; Katsifolis, J.; Cahill, L.; Roberts, A.; Nugent, K. Fabrication, modeling, and direct evanescent field measurement of tapered optical fiber sensors. J. Appl. Phys. 1999, 85, 3395.

16. Brambilla, G.; Finazzi, V.; Richardson, D.J. Ultra-low-loss optical fiber nanotapers. Opt. Express 2004, 12, 2258-2263.

17. Brambilla, G.; Jung, Y.; Renna, F. Optical fiber microwires and nanowires manufactured by modified flame brushing technique: Properties and applications. Front. Optoelectron. China 2010, 3, 61-66.

18. Ward, J.; O’Shea, D.; Shortt, B.; Morrissey, M.; Deasy, K.; Nic Chormaic, S. Heat-and-pull rig for fiber taper fabrication. Rev. Sci. Instrum. 2006, 77, 083105.

19. Ding, L.; Belacel, C.; Ducci, S.; Leo, G.; Favero, I. Ultralow loss single-mode silica tapers manufactured by a microheater. Appl. Opt. 2010, 49, 2441-2445.

20. Shi, L.; Chen, X.; Liu, H.; Chen, Y.; Ye, Z.; Liao, W.; Xia, Y. Fabrication of submicron-diameter silica fibers using electric strip heater. Opt. Express 2006, 14, 5055-5060.

21. Kenny, R.; Birks, T.; Oakley, K. Control of optical fibre taper shape. Elec. Lett. 1991, 27, 1654-1656.

22. Birks, T.A.; Li, Y.W. The shape of fiber tapers. J. Lightwave Tech. 1992, 10, 432-438.

23. Love, J.; Henry, W.; Stewart, W.; Black, R.; Lacroix, S.; Gonthier, F. Tapered single-mode fibres and devices. I. Adiabaticity criteria. IEE Proc. J. Optoelectron. 1991, 138, 343-354. 
24. Snyder, A.W.; Love, J.D. Optical Waveguide Theory; Chapman and Hall: London, UK, 1980.

25. Sagué, G.; Baade, A.; Rauschenbeutel, A. Blue-detuned evanescent field surface traps for neutral atoms based on mode interference in ultra-thin optical fibres. New J. Phys. 2008, 10, 113008.

26. Petcu-Colan, A.; Frawley, M.; Nic Chormaic, S. Tapered few-mode tapered fibers: Mode evolution during fabrication and adiabaticity. J. Nonlin. Opt. Phys. Mater. 2011, 20, 293-307.

27. Frawley, M.C.; Petcu-Colan, A.; Viet Giang, T.; Nic Chormaic, S. Higher order mode propagation in an optical nanofiber. Opt. Commun. 2012, 285, 4648-4654.

28. Bures, J.; Ghosh, R. Power density of the evanescent field in the vicinity of a tapered fiber. J. Opt. Soc. Am. A 1999, 16, 1992-1996.

29. Franson, J.D.; Jacobs, B.C.; Pittman, T.B. Quantum computing using single photons and the zeno effect. Phys. Rev. A 2004, 70, 062302.

30. Ghosh, S.; Bhagwat, A.R.; Renshaw, C.K.; Goh, S.; Gaeta, A.L.; Kirby, B.J. Low-light-level optical interactions with rubidium vapor in a photonic band-gap fiber. Phys. Rev. Lett. 2006, 97, 023603.

31. Tanji-Suzuki, H.; Chen, W.; Landig, R.; Simon, J.; Vuletić, V. Vacuum-induced transparency. Science 2011, 333, 1266-1269.

32. Le Kien, F.; Gupta, S.D.; Balykin, V.I.; Hakuta, K. Spontaneous emission of a cesium atom near a nanofiber: Efficient coupling of light to guided modes. Phys. Rev. A 2005, 72, 032509.

33. Ebbesen, T.W.; Lezec, H.J.; Ghaemi, H.F.; Thio, T.; Wolff, P.A. Extraordinary optical transmission through sub-wavelength hole arrays. Nature 1998, 391, 667-669.

34. Choi, K.Y.; Yoon, J.; Song, S.H.; Oh, C.H.; Kim, P.S. Surface-plasmon coupling in subwavelength periodic structures. Proc. SPIE 2005, 5636, 22-26.

35. Russell, L.; Gleeson, D.A.; Minogin, V.G.; Nic Chormaic, S. Spectral distribution of atomic fluorescence coupled into an optical nanofibre. J. Phys. B Atomic Mol. Opt. Phys. 2009, 42, 185006.

36. Minogin, V.G.; Nic Chormaic, S. Manifestation of the van der Waals surface interaction in the spontaneous emission of atoms into an optical nanofiber. Laser Phys. 2010, 20, 32-37.

37. Frawley, M.C.; Nic Chormaic, S.; Minogin, V.G. The van der Waals interaction of an atom with the convex surface of a nanocylinder. Phys. Scr. 2012, 85, 058103.

38. Nayak, K.P.; Melentiev, P.N.; Morinaga, M.; Kien, F.L.; Balykin, V.I.; Hakuta, K. Optical nanofiber as an efficient tool for manipulating and probing atomic fluorescence. Opt. Express 2007, 15, 5431-5438.

39. Watkins, A.; Tiwari, V.B.; Ward, J.M.; Nic Chormaic, S. Observation of Zeeman shift in the rubidium D2 line using an optical nanofiber in vapor. 2012, arXiv:1208.4708.

40. Esslinger, T.; Bloch, I.; Hänsch, T.W. Bose-Einstein condensation in a quadrupole-Ioffe-configuration trap. Phys. Rev. A 1998, 58, R2664-R2667.

41. Schlosser, N.; Reymond, G.; Protsenko, I.; Grangier, P. Sub-Poissonian loading of single atoms in a microscopic dipole trap. Nature 2001, 411, 1024-1027.

42. Paredes, B.; Widera, A.; Murg, V.; Mandel, O.; Folling, S.; Cirac, I.; Shlyapnikov, G.V.; Hansch, T.W.; Bloch, I. Tonks-Girardeau gas of ultracold atoms in an optical lattice. Nature 2004, 429, 277-281.

43. Bolpasi, V.; Grucker, J.; Morrissey, M.J.; von Klitzing, W. A gradient and offset compensated ioffe-pritchard trap for Bose-Einstein condensation experiments. J. Phys. B Atomic Mol. Opt. Phys. 2012, 45, 235301. 
44. Richmond, J.A.; Nic Chormaic, S.; Cantwell, B.P.; Opat, G.I. A magnetic guide for cold atoms. Acta Phys. Slovaca 1998, 48, 481-488.

45. Opat, G.I.; Nic Chormaic, S.; Cantwell, B.P.; Richmond, J.A. Optical elements for slowly moving neutral atoms based on magnetic fields. J. Opt. B Quant. Semiclass. Opt. 1999, 1, 415-419.

46. Denschlag, J.; Cassettari, D.; Schmiedmayer, J. Guiding neutral atoms with a wire. Phys. Rev. Lett. 1999, 82, 2014-2017.

47. Reichel, J.; Hansell, W.; Hänsch, T.W. Atomic micromanipulation with magnetic surface traps. Phys. Rev. Lett. 1999, 83, 3398-3401.

48. Kuhr, S.; Alt, W.; Schrader, D.; Muller, M.; Gomer, V.; Meschede, D. Deterministic delivery of a single atom. Science 2001, 293, 278-280.

49. Gustavson, T.L.; Chikkatur, A.P.; Leanhardt, A.E.; Gorlitz, A.; Gupta, S.; Pritchard, D.E.; Ketterle, W. Transport of Bose-Einstein condensates with optical tweezers. Phys. Rev. Lett. 2002, 88, 020401.

50. Richmond, J.A.; Cantwell, B.P.; Chormaic, S.N.; Lau, D.C.; Akulshin, A.M.; Opat, G.I. Magnetic guide for neutral atoms. Phys. Rev. A 2002, 65, 033422.

51. Kadlecek, S.; Sebby, J.; Newell, R.; Walker, T.G. Nondestructive spatial heterodyne imaging of cold atoms. Opt. Lett. 2001, 26, 137-139.

52. Turner, L.D.; Domen, K.; Scholten, R.E. Diffraction-contrast imaging of cold atoms. Phys. Rev. A 2005, 72, 031403.

53. Pappa, M.; Condylis, P.C.; Konstantinidis, G.O.; Bolpasi, V.; Lazoudis, A.; Morizot, O.; Sahagun, D.; Baker, M.; von Klitzing, W. Ultra-sensitive atom imaging for matter-wave optics. New J. Phys. 2011, 13, 115012.

54. Le Kien, F.; Balykin, V.I.; Hakuta, K. Scattering of an evanescent light field by a single cesium atom near a nanofiber. Phys. Rev. A 2006, 73, 013819.

55. Hennessy, T.; Busch, T. Creating atom-number states around tapered optical fibers by loading from an optical lattice. Phys. Rev. A 2012, 85, 053418.

56. Masalov, A.V.; Minogin, V.G. Pumping of higher-order modes of an optical nanofiber by laser excited atoms. Las. Phys. Lett. 2013, 10, 075203.

57. Sagué, G.; Vetsch, E.; Alt, W.; Meschede, D.; Rauschenbeutel, A. Cold-atom physics using ultrathin optical fibers: Light-induced dipole forces and surface interactions. Phys. Rev. Lett. 2007, 99, 163602.

58. Nayak, K.; Hakuta, K. Single atoms on an optical nanofibre. New J. Phys. 2008, 10, 053003.

59. Hughes, S. Modified spontaneous emission and qubit entanglement from dipole-coupled quantum dots in a photonic crystal nanocavity. Phys. Rev. Lett. 2005, 94, 227402.

60. Chang, D.E.; Sorensen, A.S.; Hemmer, P.R.; Lukin, M.D. Quantum optics with surface plasmons. Phys. Rev. Lett. 2006, 97, 053002.

61. Berman, P. Cavity Quantum Electrodynamics (Advances in Atomic, Molecular and Optical Physics); Academic Press: New York, NY, USA, 1994.

62. Nha, H.; Jhe, W. Cavity quantum electrodynamics for a cylinder: Inside a hollow dielectric and near a solid dielectric cylinder. Phys. Rev. A 1997, 56, 2213-2220.

63. Sondergaard, T.; Tromborg, B. General theory for spontaneous emission in active dielectric microstructures: Example of a fiber amplifier. Phys. Rev. A 2001, 64, 033812. 
64. Klimov, V.; Ducloy, M. Spontaneous emission rate of an excited atom placed near a nanofiber. Phys. Rev. A 2004, doi:10.1016/S0030-4018(02)01802-3.

65. Nayak, K.; Le Kien, F.; Morinaga, M.; Hakuta, K. Antibunching and bunching of photons in resonance fluorescence from a few atoms into guided modes of an optical nanofiber. Nat. (Lond.) Phys. Rev. A 2009, 79, 021801.

66. Landragin, A.; Courtois, J.Y.; Labeyrie, G.; Vansteenkiste, N.; Westbrook, C.I.; Aspect, A. Measurement of the van der Waals force in an atomic mirror. Phys. Rev. Lett. 1996, 77, 1464-1467.

67. Harber, D.M.; Obrecht, J.M.; McGuirk, J.M.; Cornell, E.A. Measurement of the Casimir-Polder force through center-of-mass oscillations of a Bose-Einstein condensate. Phys. Rev. A 2005, 72, 033610.

68. Le Kien, F.; Gupta, S.D.; Hakuta, K. Optical excitation spectrum of an atom in a surface-induced potential. Phys. Rev. A 2007, 75, 032508.

69. Le Kien, F.; Hakuta, K. Spontaneous radiative decay of translational levels of an atom near a dielectric surface. Phys. Rev. A 2007, 75, 013423.

70. Nayak, K.P.; Das, M.; Kien, F.L.; Hakuta, K. Spectroscopy of near-surface atoms using an optical nanofiber. Opt. Commun. 2012, 285, 4698-4704.

71. Deasy, K.; Watkins, A.; Morrissey, M.; Schmidt, R.; Nic Chormaic, S. Few Atom Detection and Manipulation using Optical Nanofibres. In Quantum Communication and Quantum Networking; Sergienko, A., Pascazio, S., Villoresi, P., Eds.; Springer: Berlin Heidelberg, Germany, 2010; Volume 36, pp. 200-209.

72. Morrissey, M.J.; Deasy, K.; Wu, Y.; Chakrabarti, S.; Nic Chormaic, S. Tapered optical fibers as tools for probing magneto-optical trap characteristics. Rev. Sci. Instrum. 2009, 80, 053102.

73. Russell, L.; Kumar, R.; Tiwari, V.; Nic Chormaic, S. Measurements on release-recapture of cold $\mathrm{Rb}-85$ atoms using an optical nanofibre in a magneto-optical trap. Opt. Commun. 2013, doi:10.1016/j.optcom.2013.07.080.

74. Kien, F.L.; Balykin, V.I.; Hakuta, K. Light-induced force and torque on an atom outside a nanofiber. Phys. Rev. A 2006, 74, 033412.

75. Hakuta, K. Single atoms on an optical nanofiber: A novel work system for slow light. Proc. SPIE 2008, 6904, 690406.

76. Letokhov, V. Nonlinear high resolution laser spectroscopy. Science 1975, 190, 344-351.

77. Spillane, S.M.; Pati, G.S.; Salit, K.; Hall, M.; Kumar, P.; Beausoleil, R.G.; Shahriar, M.S. Observation of nonlinear optical interactions of ultralow levels of light in a tapered optical nanofiber embedded in a hot rubidium vapor. Phys. Rev. Lett. 2008, 100, 233602.

78. Hendrickson, S.M.; Pittman, T.B.; Franson, J.D. Nonlinear transmission through a tapered fiber in rubidium vapor. J. Opt. Soc. Am. B 2009, 26, 267-271.

79. Hendrickson, S.M.; Lai, M.M.; Pittman, T.B.; Franson, J.D. Observation of two-photon absorption at low power levels using tapered optical fibers in rubidium vapor. Phys. Rev. Lett. 2010, 105, 173602.

80. Russell, L.; Daly, M.; Nic Chormaic, S. 1- and 2-photon absorption by laser-cooled ${ }^{85} \mathrm{Rb}$ using an optical nanofiber. AIP Conf. Proc. 2012, 1469, 82-90. 
81. Lai, M.; Franson, J.D.; Pittman, T.B. Transmission degradation and preservation for tapered optical fibers in rubidium vapor. Appl. Opt. 2013, 52, 2595-2601.

82. Balykin, V.I.; Hakuta, K.; Le Kien, F.; Liang, J.Q.; Morinaga, M. Atom trapping and guiding with a subwavelength-diameter optical fiber. Phys. Rev. A 2004, 70, 011401.

83. Le Kien, F.; Balykin, V.I.; Hakuta, K. Atom trap and waveguide using a two-color evanescent light field around a subwavelength-diameter optical fiber. Phys. Rev. A 2004, 70, 063403.

84. Dawkins, S.T.; Mitsch, R.; Reitz, D.; Vetsch, E.; Rauschenbeutel, A. Dispersive optical interface based on nanofiber-trapped atoms. Phys. Rev. Lett. 2011, 107, 243601.

85. Schneeweiss, P.; Dawkins, S.; Mitsch, R.; Reitz, D.; Vetsch, E.; Rauschenbeutel, A. A nanofiber-based optical conveyor belt for cold atoms. Appl. Phys. B 2013, 110, 279-283.

86. Reitz, D.; Rauschenbeutel, A. Nanofiber-based double-helix dipole trap for cold neutral atoms. Opt. Commun. 2012, 285, 4705-4708.

87. Le Kien, F.; Liang, J.Q.; Hakuta, K.; Balykin, V.I. Field intensity distributions and polarization orientations in a vacuum-clad subwavelength-diameter optical fiber. Opt. Commun. 2004, 242, 445-455.

88. Fatemi, F.K.; Ravets, S.; Hoffman, J.E.; Beadie, G.; Rolston, S.L.; Orozco, L.A. Higher order mode propagation in ultrathin optical fibers for atom traps. Proc. SPIE 2013, 8637, 86370X.

89. Warken, F.; Vetsch, E.; Meschede, D.; Sokolowski, M.; Rauschenbeutel, A. Ultra-sensitive surface absorption spectroscopy using sub-wavelength diameter optical fibers. Opt. Express 2007, 15, 11952-11958.

90. Garcia-Fernandez, R.; Alt, W.; Bruse, F.; Dan, C.; Karapetyan, K.; Rehband, O.; Stiebeiner, A.; Wiedemann, U.; Meschede, D.; Rauschenbeutel, A. Optical nanofibers and spectroscopy. Appl. Phys. B Las. Opt. 2011, 105, 3-15.

91. Stiebeiner, A.; Rehband, O.; Garcia-Fernandez, R.; Rauschenbeutel, A. Ultra-sensitive fluorescence spectroscopy of isolated surface-adsorbed molecules using an optical nanofiber. Opt. Express 2009, 17, 21704-21711.

92. Takiguchi, M.; Yoshikawa, Y.; Yamamoto, T.; Nakayama, K.; Kuga, T. Saturated absorption spectroscopy of acetylene molecules with an optical nanofiber. Opt. Lett. 2011, 36, 1254-1256.

93. Wiedemann, U.; Alt, W.; Meschede, D. Switching photochromic molecules adsorbed on optical microfibres. Opt. Express 2012, 20, 12710-12720.

94. Gregor, M.; Kuhlicke, A.; Benson, O. Soft-landing and optical characterization of a preselected single fluorescent particle on a tapered optical fiber. Opt. Express 2009, 17, 24234-24243.

95. Srinivasan, K.; Painter, O.; Stintz, A.; Krishna, S. Single quantum dot spectroscopy using a fiber taper waveguide near-field optic. Appl. Phys. Lett. 2007, 91, 091102.

96. Davanco, M.; Srinivasan, K. Efficient spectroscopy of single embedded emitters using optical fiber taper waveguides. Opt. Express 2009, 17, 10542-10563.

97. Davanco, M.; Srinivasan, K. Fiber-coupled semiconductor waveguides as an efficient optical interface to a single quantum dipole. Opt. Lett. 2009, 34, 2542-2544.

98. Davanco, M.; Srinivasan, K. Hybrid gap modes induced by fiber taper waveguides: Application in spectroscopy of single solid-state emitters deposited on thin films. Opt. Express 2010, 18, 10995-11007. 
99. Yalla, R.; Nayak, K.P.; Hakuta, K. Fluorescence photon measurements from single quantum dots on an optical nanofiber. Opt. Express 2012, 20, 2932-2941.

100. Yalla, R.; Le Kien, F.; Morinaga, M.; Hakuta, K. Efficient channeling of fluorescence photons from single quantum dots into guided modes of optical nanofiber. Phys. Rev. Lett. 2012, 109, 063602.

101. Fujiwara, M.; Toubaru, K.; Noda, T.; Zhao, H.-Q.; Takeuchi, S. Highly efficient coupling of photons from nanoemitters into single-mode optical fibers. Nano Lett. 2011, 11, 4362-4365.

102. Schröder, T.; Fujiwara, M.; Noda, T.; Zhao, H.-Q.; Benson, O.; Takeuchi, S. A nanodiamond-tapered fiber system with high single-mode coupling efficiency. Opt. Express 2012, 20, 10490-10497.

103. Le Kien, F.; Hakuta, K. Cavity-enhanced channeling of emission from an atom into a nanofiber. Phys. Rev. A 2009, 80, 053826.

104. Nayak, K.P.; Le Kien, F.; Kawai, Y.; Hakuta, K.; Nakajima, K.; Miyazaki, H.T.; Sugimoto, Y. Cavity formation on an optical nanofiber using focused ion beam milling technique. Opt. Express 2011, 19, 14040-14050.

105. Tian, Y.; Wang, W.; Wu, N.; Zou, X.; Wang, X. Tapered optical fiber sensor for label-free detection of biomolecules. Sensors 2011, 11, 3780-3790.

106. Yoshie, T.; Tang, L.; Su, S.-Y. Optical microcavity: Sensing down to single molecules and atoms. Sensors 2011, 11, 1972-1991.

107. Vahala, K.J. Optical microcavities. Nature 2003, 424, 839-846.

108. Ward, J.M.; O’Shea, D.G.; Shortt, B.J.; Nic Chormaic, S. Optical bistability in Er-Yb codoped phosphate glass microspheres at room temperature. J. Appl. Phys. 2007, 102, 023104.

109. Wu, Y.; Ward, J.M.; Nic Chormaic, S. Ultralow threshold green lasing and optical bistability in ZBNA microspheres. J. Appl. Phys. 2010, 107, 033103-033106.

110. Ward, J.M.; Wu, Y.; Khalfi, K.; Nic Chormaic, S. Short vertical tube furnace for the fabrication of doped glass microsphere lasers. Rev. Sci. Instrum. 2010, 81, 073106.

111. Nic Chormaic, S.; Wu, Y.; Ward, J.; Watkins, A. Fano Resonances and Electromagnetically Induced Absorption and Transparency-like Effects in Single Silica Microspheres. In Proceedings of the Frontiers in Optics 2010/Laser Science XXVI, OSA Technical Digest (CD), Rochester, NY, USA, 24-28 October 2010.

112. Watkins, A.; Ward, J.; Wu, Y.; Nic Chormaic, S. Single-input spherical microbubble resonator. Opt. Lett. 2011, 36, 2113-2115.

113. Alton, D.J.; Stern, N.P.; Aoki, T.; Lee, H.; Ostby, E.; Vahala, K.J.; Kimble, H.J. Strong interactions of single atoms and photons near a dielectric boundary. Nat. Phys. 2011, 7, 159-165.

114. Purcell, E.M. Spontaneous emission probabilities at radio frequencies. Phys. Rev. 1946, 69, 681-681.

115. Vollmer, F.; Yang, L. Label-free detection with high-q microcavities: A review of biosensing mechanisms for integrated devices. Nanophotonics 2012, 1, 267-291.

116. Armani, A.M.; Kulkarni, R.P.; Fraser, S.E.; Flagan, R.C.; Vahala, K.J. Label-free, single-molecule detection with optical microcavities. Science 2007, 317, 783-787.

117. Knight, J.C.; Cheung, G.; Jacques, F.; Birks, T.A. Phase-matched excitation of whispering-gallery-mode resonances by a fiber taper. Opt. Lett. 1997, 22, 1129-1131.

118. Cai, M.; Painter, O.; Vahala, K.J. Observation of critical coupling in a fiber taper to a silica-microsphere whispering-gallery mode system. Phys. Rev. Lett. 2000, 85, 74-77. 
119. Spillane, S.M.; Kippenberg, T.J.; Painter, O.J.; Vahala, K.J. Ideality in a fiber-taper-coupled microresonator system for application to cavity quantum electrodynamics. Phys. Rev. Lett. 2003, 91, 043902.

120. Srinivasan, K.; Painter, O. Optical fiber taper coupling and high-resolution wavelength tuning of microdisk resonators at cryogenic temperatures. Appl. Phys. Lett. 2007, 90, 031114.

121. Watkins, A.; Ward, J.; Nic Chormaic, S. Thermo-optical tuning of whispering gallery modes in Erbium: Ytterbium doped glass microspheres to arbitrary probe wavelengths. Jpn. J. Appl. Phys. 2012, 51, 052501.

122. Kakarantzas, G.; Dimmick, T.E.; Birks, T.A.; Le Roux, R.; Russell, P.S.J. Miniature all-fiber devices based on $\mathrm{CO}_{2}$ laser microstructuring of tapered fibers. Opt. Lett. 2001, 26, 1137-1139.

123. Pöllinger, M.; O’Shea, D.; Warken, F.; Rauschenbeutel, A. Ultrahigh-Q tunable whispering-gallery-mode microresonator. Phys. Rev. Lett. 2009, 103, 053901.

124. Junge, C.; O’Shea, D.; Volz, J.; Rauschenbeutel, A. Strong coupling between single atoms and nontransversal photons. Phys. Rev. Lett. 2013, 110, 213604.

125. Fam Le, K.; Hakuta, K. Intracavity electromagnetically induced transparency in atoms around a nanofiber with a pair of Bragg grating mirrors. Phys. Rev. A 2009, 79, 043813.

126. Fam Le, K.; Hakuta, K. Triggered generation of single guided photons from a single atom in a nanofiber cavity. Phys. Rev. A 2011, 83, 043801.

127. Le Kien, F.; Hakuta, K. Deterministic generation of a pair of entangled guided photons from a single atom in a nanofiber cavity. Phys. Rev. A 2011, 84, 053801.

128. Kien, F.L.; Nayak, K.P.; Hakuta, K. Nanofibers with Bragg gratings from equidistant holes. J. Mod. Opt. 2012, 59, 274-286.

129. Fam Le, K.; Hakuta, K. Translational motion of an atom in a weakly driven fiber-Bragg-grating cavity. Adv. Nat. Sci. Nanosci. Nanotechnol. 2012, 3, 025009.

130. Lindner, E.; Chojetzki, C.; Brückner, S.; Becker, M.; Rothhardt, M.; Bartelt, H. Thermal regeneration of fiber Bragg gratings in photosensitive fibers. Opt. Express 2009, 17, 12523-12531.

131. Zhang, Y.; Lin, B.; Tjin, S.C.; Zhang, H.; Wang, G.; Shum, P.; Zhang, X. Refractive index sensing based on higher-order mode reflection of a microfiber Bragg grating. Opt. Express 2010 , 18, 26345-26350.

132. Wieduwilt, T.; Brueckner, S.; Bartelt, H. High force measurement sensitivity with fiber Bragg gratings fabricated in uniform-waist fiber tapers. Meas. Sci. Tech. 2011, 22, 075201.

133. Yang, R.; Long, J.; Yan-Nan, T.; Li-Peng, S.; Jie, L.; Bai-Ou, G. High-efficiency ultraviolet inscription of Bragg gratings in microfibers. IEEE Photon. J. 2012, 4, 181-186.

134. Ding, M.; Zervas, M.N.; Brambilla, G. A compact broadband microfiber Bragg grating. Opt. Express 2011, 19, 15621-15626.

135. Liu, Y.; Meng, C.; Zhang, A.P.; Xiao, Y.; Yu, H.; Tong, L. Compact microfiber Bragg gratings with high-index contrast. Opt. Lett. 2011, 36, 3115-3117.

136. Ding, M.; Wang, P.; Lee, T.; Brambilla, G. A microfiber cavity with minimal-volume confinement. Appl. Phys. Lett. 2011, 99, 051105.

137. Feng, J.; Ding, M.; Kou, J.-L.; Xu, F.; Lu, Y.-Q. An optical fiber tip micrograting thermometer. IEEE Photon. J. 2011, 3, 810-814. 
138. Dragomir, A.; Nikogosyan, D.N.; Zagorulko, K.A.; Kryukov, P.G.; Dianov, E.M. Inscription of fiber Bragg gratings by ultraviolet femtosecond radiation. Opt. Lett. 2003, 28, 2171-2173.

139. Grobnic, D.; Mihailov, S.J.; Ding, H.M.; Smelser, C.W. Bragg grating evanescent field sensor made in biconical tapered fiber with femtosecond ir radiation. IEEE Photon. Tech. Lett. 2006, 18, $160-162$.

140. Zhao, P.; Li, Y.; Zhang, J.; Shi, L.; Zhang, X. Nanohole induced microfiber Bragg gratings. Opt. Express 2012, 20, 28625-28630.

141. Nayak, K.P.; Hakuta, K. Photonic crystal formation on optical nanofibers using femtosecond laser ablation technique. Opt. Express 2013, 21, 2480-2490.

142. Wuttke, C.; Becker, M.; Brückner, S.; Rothhardt, M.; Rauschenbeutel, A. Nanofiber Fabry-Perot microresonator for nonlinear optics and cavity quantum electrodynamics. Opt. Lett. 2012, 37, 1949-1951.

143. Sumetsky, M. Optical fiber microcoil resonator. Opt. Express 2004, 12, 2303-2316.

144. Fei, X.; Horak, P.; Brambilla, G. Optimized design of microcoil resonators. J. Lightwave Tech. 2007, 25, 1561-1567.

145. Fei, X.; Brambilla, G. Manufacture of 3-D microfiber coil resonators. IEEE Photon. Tech. Lett. 2007, 19, 1481-1483.

146. Jiang, X.; Yang, Q.; Vienne, G.; Li, Y.; Tong, L.; Zhang, J.; Hu, L. Demonstration of microfiber knot laser. Appl. Phys. Lett. 2006, 89, 143513.

147. Xiao, L.; Birks, T.A. High finesse microfiber knot resonators made from double-ended tapered fibers. Opt. Lett. 2011, 36, 1098-1100.

148. Sumetsky, M.; Dulashko, Y.; Fini, J.M.; Hale, A. Optical microfiber loop resonator. Appl. Phys. Lett. 2005, 86, 161108.

149. Sumetsky, M.; Dulashko, Y.; Fini, J.M.; Hale, A.; DiGiovanni, D.J. The microfiber loop resonator: Theory, experiment, and application. J. Lightwave Tech. 2006, 24, 242-250.

150. Yu, W.; Xu, Z.; Changlun, H.; Jian, B.; Guoguang, Y. A tunable all-fiber filter based on microfiber loop resonator. Appl. Phys. Lett. 2008, 92, 191112.

151. Chen, Z.; Hsiao, V.K.S.; Li, X.; Li, Z.; Yu, J.; Zhang, J. Optically tunable microfiber-knot resonator. Opt. Express 2011, 19, 14217-14222.

152. Chen, G.Y.; Lee, T.; Zhang, X.L.; Brambilla, G.; Newson, T.P. Temperature compensation techniques for resonantly enhanced sensors and devices based on optical microcoil resonators. Opt. Commun. 2012, 285, 4677-4683.

153. Sulaiman, A.; Harun, S.W.; Ahmad, F.; Norizan, S.F.; Ahmad, H. Electrically tunable microfiber knot resonator based erbium-doped fiber laser. IEEE J. Quantum Electron. 2012, 48, 443-446.

154. Sulaiman, A.; Harun, S.W.; Ahmad, H. Thermally tunable microfiber knot resonator based erbium-doped fiber laser. Opt. Commun. 2012, 285, 4684-4687.

155. Jung, Y.M.; Brambilla, G.; Richardson, D.J. Polarization-maintaining optical microfiber. Opt. Lett. 2010, 35, 2034-2036.

(C) 2013 by the authors; licensee MDPI, Basel, Switzerland. This article is an open access article distributed under the terms and conditions of the Creative Commons Attribution license (http://creativecommons.org/licenses/by/3.0/). 